\title{
On the Proof of the Positive Mass Conjecture in General Relativity
}

\author{
Richard Schoen and Shing-Tung Yau
}

\begin{abstract}
Let $M$ be a space-time whose local mass density is non-negative everywhere. Then we prove that the total mass of $M$ as viewed from spatial infinity (the ADM mass) must be positive unless $M$ is the flat Minkowski space-time. (So far we are making the reasonable assumption of the existence of a maximal spacelike hypersurface. We will treat this topic separately.) We can generalize our result to admit wormholes in the initial-data set. In fact, we show that the total mass associated with each asymptotic regime is nonnegative with equality only if the space-time is flat.
\end{abstract}

\section{Introduction}

This is the second part of our paper on scalar curvature of a three-dimensional manifold and its relation to general relativity. The problem in general relativity that we address is the following: An isolated gravitating system having nonnegative local mass density must have non-negative total mass, measured gravitationally at spatial infinity.

Mathematically, the positive mass conjecture can be described as follows: Let $N$ be a three dimensional Riemannian manifold with metric tensor $g_{i j}$. Then an initial set consists of $N$ and a symmetric tensor field $h_{i j}$ so that $\mu \geqq\left|\sum_{a} J^{a} J_{a}\right|^{1 / 2}$ where $\mu$ and $J$ are defined by

$$
\begin{aligned}
& \mu=\frac{1}{2}\left(R-\sum_{a, b} h^{a b} h_{a b}+\left(\sum_{a} h_{a}^{a}\right)^{2}\right), \\
& J^{a}=\nabla_{b}\left[h^{a b}-\left(\sum_{c} h_{c}^{c}\right) g^{a b}\right]
\end{aligned}
$$

where $R$ is the scalar curvature of our metric.

If $N$ is a spacelike hypersurface in a space time so that $g_{i j}$ is the induced metric and $h_{i j}$ is the second fundamental form, then the above condition says that the apparent energy-momentum of the matter be timelike. 
An initial-data set will be said to be asymptotically flat if for some compact $C$, $N \backslash C$ consists of a finite number of components $N_{1}, \ldots, N_{k}$ such that each $N_{i}$ is diffeomorphic to the complement of a compact set in $R^{3}$. Under such diffeomorphism, the metric tensor will be required to be written in the following form

$$
d s^{2}=\left(1+\frac{M}{2 r}\right)^{4}\left(\sum_{i}\left(d x^{i}\right)^{2}\right)+\sum_{i, j} p_{i j} d x^{i} d x^{j}
$$

where

$$
\begin{aligned}
p_{i j} & =O\left(\frac{1}{r^{2}}\right), \\
\nabla p_{i j} & =O\left(\frac{1}{r^{3}}\right)
\end{aligned}
$$

and

$$
\nabla \nabla p_{i j}=O\left(\frac{1}{r^{4}}\right) .
$$

The components of $h_{i j}$ will also be required to be of order $O\left(\frac{1}{r^{2}}\right)$.

The number $M$ (Arnowitt, Deser and Misner [1], Geroch [9]) is called the mass of the end $N_{i}$. This definition is motivated by the observation that the spatial Schwarzschild metric can be written asymptotically in the previous form so that the number $M$ is precisely the Schwarzschild mass. From now on, we shall call $N_{i}$ an "end" of $N$ and we denote the total mass of $N_{i}$ by $M_{i}$.

In this formulation, the (generalized) positive mass conjecture (Arnowitt et al. [1], Brill and Deser [3], Geroch [2]) states that for an asymptotically flat initial data set, each end has non-negative total mass. If one of the ends has zero total mass, then the initial data set is flat in the sense that the curvature tensor vanishes and the second fundamental form $h_{i j}$ is trivial.

In this paper, we will settle the major case of the conjecture assuming $\sum_{a} h_{a}^{a}=0$. The most general case will be discussed in a forthcoming paper.

There have been several contributions on this problem prior to our work. (We learned most of these from the excellent survey articles of Geroch [2] and Choquet-Bruhat, Fisher and Marsden [4].) In 1959, Brill settled the problem in case $\sum_{a} h_{a}^{a}=0$ and the data respect an axial symmetry. In 1968, Brill and Deser [3] showed the conjecture is true up to second order perturbations from flat data. This last result was greatly improved by Choquet-Bruhat and Marsden [5] to the effect that the conjecture is true if the data is close enough to the flat data in a certain smooth norm. In the Stanford conference in differential geometry, Geroch divided the conjecture into several special cases. One case had a direct appeal to the geometers. This case says that if a metric has non-negative scalar curvature in $R^{3}$ and if the metric is euclidean outside a compact set, then the metric is flat. In her thesis in 1977, Leite was able to settle this case under the assumption that the 
manifold can be isometrically embedded into $R^{4}$. In 1976, Jang [6] was also able to settle the conjecture if the metric $g_{i j}$ is flat. Finally the conjecture was also known if the data is sperically symmetric (Leibovitz and Israel [7], Misner [8], Jang [6]). However, none of these methods had been carried out to cover the case that we deal with. (It should also be mentioned that Deser had a proof for the supergravity setting and Geroch had an argument to settle the conjecture assuming some statement that remains to be proved.)

The basic idea of our proof is quite simple. It is basically geometric in nature which enables us to deal with the case where the manifold is not diffeomorphic to $R^{3}$. While there are more details to be carried out in this paper, the basic ideas are already in our previous paper.

For simplicity, let us assume the manifold is diffeomorphic to $R^{3}$. Then assuming the mass is negative we construct a complete surface embedded in $R^{3}$ whose area is minimal among all compactly supported deformations of the surface. By using the second variation formula, we prove that the surface is topologically the plane. As in the previous paper, we plan to use the Gauss-Bonnet theorem to arrive at a contradiction. However, as the surface is non-compact, there are technical troubles involved which we are able to overcome. These arguments provide a proof that the total mass is non-negative. If the total mass is zero, then we have a way to reduce it to the previous case unless the Ricci tensor is identically zero. Since $M$ is three-dimensional, Ricci flat implies flat and the reduction finishes the proof of the theorem.

\section{Statement of Results}

The theorems in this paper deal with asymptotically flat metrics on 3-manifolds. Let $N$ be an oriented three-dimensional manifold (with or without boundary) which has the property that there exists a compact subset $K$ of $N$ so that $N \backslash K$ consists of a finite number of components $N_{1}, N_{2}, \ldots, N_{r}$ with each $N_{k}$ being diffeomorphic to $\mathbb{R}^{3}$ minus a ball. We call the $N_{k}$ ends of $N$. We suppose that $N$ is a manifold of smoothness class $C^{6}$. Let $d s^{2}$ be a $C^{5}$ positive definite metric on $N$. We say that $d s^{2}$ is asymptotically flat if each boundary component of $N$ has positive mean curvature with respect to the outward unit normal, and on each $N_{k}$ there is a coordinate system $x^{1}, x^{2}, x^{3}$ in which $d s^{2}$ has the expansion $d s^{2}=\sum_{i, j=1}^{3} g_{i j} d x^{i} d x^{j}$ with the $g_{i j}$ satisfying the following inequalities for some $k_{1}, k_{2}$, $k_{3}$ positive constants.

$$
\begin{gathered}
g_{i j}=\left(1+\frac{M}{2 r}\right)^{4} \delta_{i j}+h_{i j}, \quad\left|h_{i j}\right| \leqq \frac{k_{1}}{1+r^{2}}, \\
\left|\partial h_{i j}\right| \leqq \frac{k_{2}}{1+r^{3}}, \quad\left|\partial \partial h_{i j}\right| \leqq \frac{k_{3}}{1+r^{4}}
\end{gathered}
$$

where $r=\left(\sum_{i=1}^{3}\left(x^{i}\right)^{2}\right)^{1 / 2}$ and $\partial$ is the Euclidean gradient. The number $M=M_{k}$ is the total mass of $N_{k}$. We note that (1.1) implies that the Christoffel symbols 
$\Gamma_{j k}^{i}=O\left(1 / r^{2}\right)$ and the curvature tensor is $O\left(1 / r^{3}\right)$ as $r \rightarrow \infty$. Let $R$ be the scalar curvature function for $d s^{2}$. We now state our first theorem.

Theorem 1. Let $d s^{2}$ be an asymptotically flat metric on an oriented 3-manifold $N$. If $R \geqq 0$ on $N$, then the total mass of each end is nonnegative.

Our next result concerns the case when total mass on one end is zero. In this case we wish to show that $N$ is flat. In order to prove this we need to add the following assumption to (1.1)

$$
\left|\partial \partial \partial h_{i j}\right|+\left|\partial \partial \partial \partial h_{i j}\right|+\left|\partial \partial \partial \partial \partial h_{i j}\right| \leqq \frac{k_{4}}{1+r^{5}}
$$

for a positive constant $k_{4}$.

Theorem 2. Let $N$ be an oriented 3-manifold having an asymptotically flat metric $d s^{2}$. Suppose for some end $N_{k},(1.2)$ is satisfied and the total mass of $N_{k}$ is zero. If $R \geqq 0$ on $N$, then $d s^{2}$ is flat. In fact, $N$ is isometric to $\mathbb{R}^{3}$ with the standard metric.

\section{Proof of Theorem 1}

Throughout this section we work on a fixed end $N_{k}$, and suppose that $x^{1}, x^{2}, x^{3}$ are asymptotically flat coordinates on $N_{k}$. Suppose these coordinates describe $N_{k}$ on $\mathbb{R}^{3} \backslash B_{\sigma_{0}}(0)$, where $B_{\sigma_{0}}(0)=\left\{|x|<\sigma_{0}\right\}$ and $r=|x|$ denotes the Euclidean length of $x=\left(x^{1}, x^{2}, x^{3}\right)$. We denote the total mass of $N_{k}$ by $M$, omitting reference to $k$. We will suppose that $M<0$ and $R \geqq 0$ in contradiction to Theorem 1 . The proof then involves three steps, the first allowing us to assume $R>0$ outside a compact subset of $\mathbb{R}^{3} \backslash B_{\sigma_{0}}(0)$, the second is to use the assumption $M<0$ to prove the existence of a complete area minimizing surface, and third to use second variation arguments to show that this is impossible if $R \geqq 0$.

Step 1. If $d s^{2}$ is asymptotically flat on $N$ with $R \geqq 0$, and with the total mass of $N_{k}$ negative, then there is an asymptotically flat metric $d \tilde{s}^{2}$ conformally equivalent to $d s^{2}$ having $\tilde{R} \geqq 0$ on $N, \tilde{R}>0$ outside a compact subset of $N_{k}$, and having negative total mass for $N_{k}$.

Proof. Let $\mathbb{R}^{3} \backslash B_{\sigma_{0}}(0)$ represent $N_{k}$ as described above. Let $\Delta$ be the Laplace operator on functions, so that for a function $\varphi$ on $\mathbb{R}^{3} \backslash B_{\sigma_{0}}(0)$

$$
\Delta \varphi=\frac{1}{\sqrt{g}} \sum_{i, j=1}^{3} \frac{\partial}{\partial x^{i}}\left(\sqrt{g} g^{i j} \frac{\partial \varphi}{\partial x^{j}}\right)
$$

where as usual, $g=\operatorname{det}\left(g_{i j}\right)$ and $\left(g^{i j}\right)=\left(g_{i j}\right)^{-1}$. We calculate the asymptotic expansion of $\Delta_{r}^{1}$ on $\mathbb{R}^{3} \backslash B_{\sigma_{0}}(0)$ using (1.1). We see that

$$
\begin{aligned}
\Delta \frac{1}{r} & =\sum_{i=1}^{3} \frac{\partial}{\partial x^{i}}\left(\left(1+\frac{M}{2 r}\right)^{2} \frac{\partial}{\partial x^{i}}\left(\frac{1}{r}\right)\right)+O\left(1 / r^{5}\right) \\
& =\left(1+\frac{M}{2 r}\right) \frac{M}{r^{4}}+O\left({ }^{1} / r^{5}\right) \\
& =\frac{M}{r^{4}}+O\left(1 / r^{5}\right) .
\end{aligned}
$$


It follows that there is a number $\sigma>\sigma_{0}$ so that

$$
\Delta \frac{1}{r}<0 \quad \text { for } \quad r \geqq \sigma .
$$

Choose $t_{0}=-\frac{M}{8 \sigma_{0}}$, and let $\zeta(t)$ be a $C^{5}$ function which satisfies

$$
\begin{aligned}
& \zeta(t)=\left\{\begin{array}{cc}
t & \text { for } \quad t<t_{0} \\
\frac{3 t_{0}}{2} & \text { for } \quad t>2 t_{0},
\end{array}\right. \\
& \zeta^{\prime}(t) \geqq 0, \quad \zeta^{\prime \prime}(t) \leqq 0 \text { for } t \in(0, \infty) .
\end{aligned}
$$

Define a $C^{5}$ function $\varphi: N \rightarrow \mathbb{R}$ by

$$
\begin{gathered}
\varphi=1+\frac{3 t_{0}}{2} \quad \text { on } \quad N \backslash N_{k}, \\
\varphi(x)=1+\zeta\left(-\frac{M}{4 r}\right) \quad \text { on } \quad \mathbb{R}^{3} \backslash B_{\sigma_{0}}(0)=N_{k} .
\end{gathered}
$$

From (2.1) and (2.2) we see that

$$
\Delta \varphi \leqq 0 \text { on } N \text {, and } \Delta \varphi<0 \text { for } r>2 \sigma .
$$

We now define a new metric

$$
d \tilde{s}^{2}=\varphi^{4} d s^{2} .
$$

The metric $d \tilde{s}^{2}$ is asymptotically flat since on all ends other than $N_{k}$ it is a constant multiple of $d s^{2}$, and on $N_{k}$ we have

$$
\begin{aligned}
\tilde{g}_{i j} & =\left(1-\frac{M}{4 r}\right)^{4}\left(1+\frac{M}{2 r}\right)^{4} \delta_{i j}+O\left(1 / r^{2}\right) \\
& =\left(1+\frac{M}{4 r}\right)^{4} \delta_{i j}+O\left(1 / r^{2}\right) .
\end{aligned}
$$

Thus the new mass of $N_{k}$ is $\tilde{M}=\frac{M}{2}<0$. The well-known formula for the scalar curvature $\tilde{R}$ is

$$
\tilde{R}=\varphi^{-5}[-8 \Delta \varphi+R \varphi] .
$$

Thus (2.3) implies that $\tilde{R} \geqq 0$ on $N$ and $\tilde{R}>0$ for $r>2 \sigma$ on $N_{k}$. This concludes the proof of Step 1.

We replace our original metric $d s^{2}$ by $d \tilde{s}^{2}$ but maintain the notation $d s^{2}$, so that we are assuming $R \geqq 0$ on $N, R>0$ outside a compact subset of $N_{k}$, and $M<0$.

Step 2. There exists a complete area minimizing (relative to $d s^{2}$ ) surface $S$ properly imbedded in $N$ so that $S \cap\left(N \backslash N_{k}\right)$ is compact, and $S \cap N_{k}$ lies between two parallel Euclidean 2-planes in the 3-space defined by $x^{1}, x^{2}, x^{3}$. 
Proof. Let $\sigma>2 \sigma_{0}$, and let $C_{\sigma}$ be the circle of Euclidean radius $\sigma$ centered at 0 in the $x^{1} x^{2}$-plane. Let $S_{\sigma}$ be the smooth imbedded oriented surface of least $d s^{2}$-area among all competing surfaces regardless of topological type having boundary curve $C_{\sigma}$. A discussion of this known existence result is given in the Appendix to this paper. We wish to extract a sequence $\sigma_{i} \rightarrow \infty$ so that $S_{\sigma_{i}}$ converges to the required surface $S$.

We first show that there is a compact subset $K_{0} \subseteq N$ so that we have

$$
\mathrm{S}_{\sigma} \cap\left(N \backslash N_{k}\right) \subseteq K_{0} \quad \text { for every } \quad \sigma>2 \sigma_{0} .
$$

That is, we show that the $S_{\sigma}$ cannot run to infinity in an end other than $N_{k}$. To see this, let $N_{k^{\prime}}$ be another end, with asymptotically flat coordinate system $y^{1}, y^{2}, y^{3}$ associating $N_{k^{\prime}}$ with $\mathbb{R}^{3} \backslash B_{\tau_{0}}(0)$ where $B_{\tau_{0}}(0)=\left\{y:|y|<\tau_{0}\right\}$. In this coordinate system, the metric $d s^{2}$ has the form $d s^{2}=\sum_{i, j=1}^{3} g_{i j}^{\prime} d y^{i} d y^{j}$ with $g_{i j}^{\prime}$ satisfying (1.1). We calculate the covariant hessian of the function $|y|^{2}$, that is $D_{i j}|y|^{2}=\frac{\partial^{2}|y|^{2}}{\partial y^{i} \partial y^{j}}$ $-D_{\frac{\partial}{\partial y^{2}}} \frac{\partial}{\partial y^{j}}\left(|y|^{2}\right)$. By $(1.1)$ we see

$$
D_{i j}|y|^{2}=2 \delta_{i j}+O(1 /|y|) \text { as }|y| \rightarrow \infty
$$

where $\delta_{i j}$ is the Kronecker delta. In particular, we see that there exists $\tau_{1}>\tau_{0}$ so that the function $|y|^{2}$ is a convex function for $|y| \geqq \tau_{1}$. Since $\partial S_{\sigma}=C_{\sigma}$ which lies in $N_{k}$, we may apply the maximum principle to conclude that

$$
S_{\sigma} \cap N_{k^{\prime}} \subseteq B_{\tau_{1}}(0) \text {. }
$$

Since $N_{k^{\prime}}$ was any end of $N$ other than $N_{k}$, we have established (2.4).

We now analyze the behavior of $S_{\sigma} \cap N_{k}$. In fact, we bound the height of $S_{\sigma} \cap N_{k}$ in the $x^{3}$ direction. For any $h>0$, we let

$$
E_{h}=\left\{x \in \mathbb{R}^{3}:\left|x^{3}\right| \leqq h\right\} .
$$

We show that there exists a number $h>\sigma_{0}$ so that

$$
N_{k} \cap S_{\sigma} \subseteq E_{h} \text { for all } \sigma>2 \sigma_{0} .
$$

To accomplish this, we again use a maximum principle, this time for the function $x^{3}$ restricted on $S_{\sigma} \cap N_{k}$. We must first compute the asymptotic behavior of the covariant hessian of $x^{3}$ on $N_{k}$. If $D$ is the Riemannian connection for $d s^{2}$, define $\Gamma_{i j}^{l}$ by

$$
D_{\frac{\partial}{\partial x^{l}}} \frac{\partial}{\partial x^{j}}=\sum_{l=1}^{3} \Gamma_{i j}^{l} \frac{\partial}{\partial x^{l}} .
$$

Then $\Gamma_{i j}^{l}$ has the following expression in terms of $d s^{2}$

$$
\Gamma_{i j}^{l}=\frac{1}{2} g^{l m}\left(\frac{\partial g_{i m}}{\partial x^{j}}+\frac{\partial g_{j m}}{\partial x^{i}}-\frac{\partial g_{i j}}{\partial x^{m}}\right) .
$$

The hessian of any function $\varphi$ is given by

$$
D_{i j} \varphi=\frac{\partial^{2} \varphi}{\partial x^{i} \partial x^{j}}-D_{\frac{\partial}{\partial x^{i}}} \frac{\partial}{\partial x^{j}}(\varphi)
$$


By direct calculation using (1.1) and (2.6) we have

$$
D_{i j} x^{3}=-\Gamma_{i j}^{3}=\frac{M x^{j}}{r^{3}} \delta_{i 3}+\frac{M x^{i}}{r^{3}} \delta_{j 3}-\frac{M x^{3}}{r^{3}} \delta_{i j}+O\left(1 / r^{3}\right) .
$$

Let $\bar{h}$ be the maximum for $x^{3}$ on $S_{\sigma} \cap N_{k}$, and suppose this maximum occurs at the point $x_{0} \in S_{\sigma}$. If $\bar{h} \leqq \sigma_{0}$, we have established (2.5). So suppose $\bar{h}>\sigma_{0}$. The tangent space to $S_{\sigma}$ at $x_{0}$ is then spanned by $\frac{\partial}{\partial x^{1}}\left(x_{0}\right), \frac{\partial}{\partial x^{2}}\left(x_{0}\right)$. Let $v_{1} v_{2}$ be tangent vector fields to $S_{\sigma}$ defined in a neighborhood of $x_{0}$ and satisfying $v_{i}\left(x_{0}\right)=\frac{\partial}{\partial x^{i}}\left(x_{0}\right)$ for $i=1,2$. Let $\left(q_{i j}\right)_{1 \leqq i, j \leqq 2}$ be the restriction of $d s^{2}$ to $S$ in terms of the base field $v_{1}, v_{2}$. Let $\nabla$ be the induced connection on $S_{\sigma}$, and note

$$
\begin{aligned}
v_{i} v_{j} x^{3}-\nabla_{v_{l}} v_{j}\left(x^{3}\right)= & v_{i} v_{j} x^{3}-D_{v_{i}} v_{j}\left(x^{3}\right) \\
& +\left\langle D_{v_{l}} v_{j}, v\right\rangle v\left(x^{3}\right)
\end{aligned}
$$

where $v$ is the unit normal field of $S_{\sigma}$. Evaluating at the point $x_{0}$ we have

$$
\nabla_{i j} x^{3}=D_{i j} x^{3}+h_{i j} v\left(x^{3}\right)
$$

where $h_{i j}=\left\langle D_{v_{i}} v_{j}, v\right\rangle\left(x_{0}\right)$ is the second fundamental form. Contracting with respect to $\left(q_{i j}\right)$ we have

$$
\sum_{i, j=1}^{2} q^{i j} \nabla_{i j} x^{3}=\sum_{i, j=1}^{2} q^{i j} D_{i j} x^{3}+\sum_{i, j=1}^{2} q^{i j} h_{i j} v\left(x^{3}\right) .
$$

Since $S_{\sigma}$ is minimal we have $\sum_{i, j=1}^{2} q^{i j} h_{i j}=0$, so applying (2.7) we see

$$
\sum_{i, j=1}^{2} q^{i j} \nabla_{i j} x^{3}=-\frac{2 M \bar{h}}{r^{3}}+O\left(1 / r^{3}\right)
$$

Since $M<0$, we see that $\bar{h}$ sufficiently large implies that $\sum_{i, j=1}^{2} q^{i j} \nabla_{i j} x^{3}>0$ at $x_{0}$ contradicting the fact that $x^{3}$ attains a maximum there. A similar argument gives a lower bound on $\left.x^{3}\right|_{S_{\sigma \cap N_{k}}}$, and we have established (2.5)

Now, let $\varrho>2 \sigma_{0}$ and define the set

$$
A_{\varrho}=\left(N \backslash N_{k}\right) \cup\left\{x:|x| \geqq \sigma_{0},\left(x^{1}\right)^{2}+\left(x^{2}\right)^{2} \leqq \varrho^{2}\right\} .
$$

For any $\sigma>\varrho,(2.4)$ and (2.5) imply

$$
S_{\sigma} \cap A_{\varrho} \subseteq\left(K_{0} \cup E_{h}\right) \cap A_{\varrho}
$$

which is a compact subset of $N$. We now quote a local interior regularity estimate for area minimizing surfaces which is discussed in the Appendix.

(2.1) Regularity Estimate. Let $U_{r}(x)$ denote the geodesic ball of radius $r$ about $x \in N$. There exists a number $r_{0}>0$ so that for any point $x_{0} \in S_{\sigma}$ with $U_{r_{0}}\left(x_{0}\right) \cap C_{\sigma}=\emptyset$, it is true that $S_{\sigma} \cap U_{r_{0}}\left(x_{0}\right)$ can be written as the graph of a $C^{3}$ function $f_{\sigma}$ over the tangent plane to $S_{\sigma}$ in a normal coordinate system on $U_{r_{0}}\left(x_{0}\right)$. 
Moreover, there is a constant $c_{1}$ depending only on $\left(N, d s^{2}\right)$ which bounds all derivatives of $f_{\sigma}$ up to order three in $U_{r_{0}}\left(x_{0}\right)$. (Note that both $r_{0}$ and $c_{1}$ are independent of $\sigma$.)

It then follows from (2.8) and the Regularity Estimate that we can choose a sequence $\sigma_{i}^{(\varrho)} \rightarrow \infty$ so that $S_{\sigma_{i}^{(\ell)}} \cap A_{\varrho}$ converges in $C^{2}$ topology. Since this can be done for any $\varrho>2 \sigma_{0}$, we can take a sequence $\varrho_{j} \rightarrow \infty$ and by extracting a diagonal sequence we find a sequence $\sigma_{i} \rightarrow \infty$ so that $S_{\sigma_{1}} \rightarrow S$, an imbedded $C^{2}$-surface, uniformly in $C^{2}$ norm on compact subsets of $N$. The surface $S$ is properly imbedded by (2.8), and is clearly area minimizing on any compact subset of $N$. From (2.4) we have $S \cap\left(N \backslash N_{k}\right) \subset K_{0}$ and hence $S \cap\left(N \backslash N_{k}\right)$ is compact. From (2.5) we have $S \cap N_{k} \subseteq E_{h}$ which is the region between two parallel 2-plane in $\mathbb{R}^{3}$. This completes the proof of Step 2.

The final step in the proof of Theorem 1 is to use the condition on the scalar curvature to derive a contradiction.

Step 3. The surface $S$ constructed in Step 2 cannot exist.

Proof. For any $\sigma \geqq \sigma_{0}$, let $S_{(\sigma)}$ be the set

$$
S_{(\sigma)}=\left[S \cap\left(N \backslash N_{k}\right)\right] \cup\left[S \cap B_{\sigma}(0)\right] .
$$

The $S_{(\sigma)}$ form an exhaustion of $S$, and we can see

$$
\text { Area }\left(S_{(\sigma)}\right) \leqq C_{2} \sigma^{2}
$$

for a constant $C_{2}$ independent of $\sigma \geqq \sigma_{0}$. To prove (2.9), we note that if $S$ has transverse intersection with $\partial B_{\sigma}(0)$ then this intersection is a union of oriented $C^{2}$ Jordan curves on $\partial B_{\sigma}(0)$ which bound $S_{(\sigma)}$. It follows that these curves bound a domain $\Omega \leqq \partial B_{\sigma}(0)$. Thus we have $\partial S_{(\sigma)}=\partial \Omega$, so we can apply the area minimizing property of $S$ to conclude that

$$
\text { Area }\left(S_{(\sigma)}\right) \leqq \text { Area }(\Omega) \leqq \text { Area }\left(\partial B_{\sigma}(0)\right) \text {. }
$$

Since (1.1) implies that $d s^{2}$ is uniformly equivalent to the Euclidean metric on $\mathbb{R}^{3} \backslash B_{\sigma_{0}}(0)$, (2.9) follows for those $\sigma>\sigma_{0}$ for which $S \cap \partial B_{\sigma}(0)$ is transverse. Since this is true except for $\sigma$ in a set of measure zero, (2.9) follows for any $\sigma \geqq \sigma_{0}$ by approximation.

We can use (2.9) to bound the integrals of certain functions on $S$. For $a>0$ we have

$$
\begin{aligned}
\int_{S} \frac{1}{1+r^{a}} & =\int_{S_{\left(\sigma_{0}\right)}} \frac{1}{1+r^{a}}+\int_{\sigma_{0}}^{\infty}\left(\frac{d}{d t} \int_{S_{t}} \frac{1}{1+r^{a}}\right) d t \\
& \leqq \operatorname{Area}\left(S_{\left(\sigma_{0}\right)}\right)+\int_{\sigma_{0}}^{\infty} \frac{1}{1+t^{a}}\left(\frac{d}{d t} \operatorname{Area}\left(S_{(t)}\right)\right) d t .
\end{aligned}
$$

If $a>2$, we can integrate by parts and apply (2.9) to get

$$
\begin{aligned}
\int_{S} \frac{1}{1+r^{a}} & \leqq c_{2} \sigma_{0}^{2}+a \int_{\sigma_{0}}^{\infty} \frac{t^{a-1}}{\left(1+t^{a}\right)^{2}} \operatorname{Area}\left(S_{(t)}\right) d t \\
& \leqq c_{2} \sigma_{0}^{2}+c_{2} a \int_{\sigma_{0}}^{\infty} \frac{t^{a+1}}{\left(1+t^{a}\right)^{2}} d t .
\end{aligned}
$$


It follows that we have

$$
\int_{S} \frac{1}{1+r^{a}}<\infty \quad \text { whenever } \quad a>2 .
$$

Applying similar reasoning, we have for $\sigma_{0}<\sigma_{1}<\sigma_{2}$,

$$
\int_{S_{\left(\sigma_{2}\right)} \backslash S_{\left(\sigma_{1}\right)}} \frac{1}{r^{2}} \leqq 2 C_{2} \log \sigma_{2} / \sigma_{1} .
$$

We now introduce the second variation inequality for $S$. This inequality expresses the fact that up to second order $S$ has smallest area in a one-parameter compactly supported deformation of $S$. Let $e_{1}, e_{2}, e_{3}$ be orthonormal (with respect to $d s^{2}$ ) vector fields defined locally on $N$. We use the notation

$$
K_{i j}=\text { sectional curvature of the section }\left\{e_{i}, e_{j}\right\} \text {. }
$$

The Ricci tensor can then be written

$$
\operatorname{Ric}\left(e_{i}\right)=\sum_{j=1}^{3} K_{i j}
$$

where we let $K_{i i}=0$. The scalar curvature $R$ is then given by

$$
R=K_{12}+K_{13}+K_{23} \text {. }
$$

Let $v$ be the unit normal vector field of $S$, and choose a frame $e_{1}, e_{2}, e_{3}=v$ adapted to $S$. Let $A$ denote the second fundamental form of $S$, i.e. the matrix in terms of $e_{1}, e_{2}$ is

$$
h_{i j}=\left\langle D_{e_{i}} \nu, e_{j}\right\rangle \text {. }
$$

It is well-known that $A$ is a symmetric quadratic tensor on $S$. We let $\|A\|^{2}$ denote the length of $A$ with respect to $d s^{2}$, i.e.

$$
\|A\|^{2}=\sum_{i, j=1}^{2} h_{i j}^{2} .
$$

The condition that $S$ is a minimal surface is

$$
\operatorname{Trace}(A)=h_{11}+h_{22}=0 \text {. }
$$

The second variation inequality (see [10]) for $S$ is

$$
\int_{S} f\left[\Delta f+\left(\operatorname{Ric}(v)+\|A\|^{2}\right) f\right] \leqq 0
$$

for any $C^{2}$ function $f$ with compact support on $S$. After integration by parts we see

$$
\int_{S}\left(\operatorname{Ric}(v)+\|A\|^{2}\right) f^{2} \leqq \int_{S}\|\nabla f\|^{2}
$$

for any $C^{2}$ function $f$ with compact support on $S$. By approximation we see easily that (2.13) holds for any Lipschitz function $f$ with compact support on $S$. The Gauss curvature equation expresses the Gauss curvature $K$ of $S$ as

$$
K=K_{12}+h_{11} h_{22}-h_{12}^{2} \text {. }
$$


Applying (2.12) and the symmetry of $A$ gives

$$
\frac{1}{2}\|A\|^{2}=K_{12}-K \text {. }
$$

Putting this into (2.13) gives

$$
\int_{S}\left(\operatorname{Ric}(v)+K_{12}-K+\frac{1}{2}\|A\|^{2}\right) f^{2} \leqq \int_{S}\|\nabla f\|^{2} .
$$

That is, we have

$$
\int_{S}\left(R-K+\frac{1}{2}\|A\|^{2}\right) f^{2} \leqq \int_{S}\|\nabla f\|^{2} .
$$

We now choose a suitable cutoff function for $f$ in our inequalities. For $\sigma>\sigma_{0}$ define a function $\varphi$ by

$$
\varphi=\left\{\begin{array}{ccc}
1 & \text { on } & S_{(\sigma)} \\
\frac{\log \frac{\sigma^{2}}{r}}{\log \sigma} & \text { on } & S_{\left(\sigma^{2}\right)} \backslash S_{(\sigma)} \\
0 & \text { outside } & S_{\left(\sigma^{2}\right)}
\end{array} .\right.
$$

Let $g$ be a Lipschitz function on $S$ satisfying $|g| \leqq 1$ and $g=1$ outside a compact subset of $S$. Setting $f=\varphi g$ in (2.13) and applying the Schwarz inequality gives

$$
\begin{aligned}
\int_{S}\left(\operatorname{Ric}(v)+\|A\|^{2}\right) \varphi^{2} g^{2} & \leqq 2 \int_{S} g^{2}\|\nabla \varphi\|^{2}+2 \int_{S} \varphi^{2}\|\nabla g\|^{2} \\
& \leqq \frac{2}{(\log \sigma)^{2}} \int_{S_{\left(\sigma^{2}\right)} \backslash S_{(\sigma)}} \frac{\|\nabla r\|^{2}}{r^{2}}+2 \int_{S} \varphi^{2}\|\nabla g\|^{2} .
\end{aligned}
$$

Because of (1.1), there is a constant $C_{3}$ with $\|\nabla r\|^{2} \leqq C_{3}$. Thus our inequality implies by rearranging and using the definition of $\varphi$ and $g$

$$
\int_{S_{(\sigma)}}\|A\|^{2} g^{2} \leqq \frac{2 C_{3}}{(\log \sigma)^{2}} \int_{S_{\left(\sigma^{2}\right)} \backslash S_{(\sigma)}} \frac{1}{r^{2}}+2 \int_{S}\|\nabla g\|^{2}+\int_{S}|\operatorname{Ric}(v)| g^{2}
$$

Applying (2.11) we have

$$
\int_{S_{(\sigma)}}\|A\|^{2} g^{2} \leqq 2 C_{2} C_{3}(\log \sigma)^{-1}+2 \int_{S}\|\nabla g\|^{2}+\int_{S}|\operatorname{Ric}(v)| g^{2} .
$$

Letting $\sigma \rightarrow \infty$ we conclude

$$
\int_{S}\|A\|^{2} g^{2} \leqq 2 \int_{S}\|\nabla g\|^{2}+\int_{S}|\operatorname{Ric}(v)| g^{2}
$$

for any Lipschitz $g$ with $|g| \leqq 1, g \equiv 1$ outside a compact subset of $S$. By (1.1), we have $\operatorname{Ric}(v)=O\left(1 / r^{3}\right)$, so choosing $g \equiv 1$ on $S$ and applying (2.10) and $a=3$ we conclude from (2.16) that $\int_{S}\|A\|^{2}<\infty$. (The formula (2.16) with $g \neq 1$ will be used later.) 
Formula (2.14) implies that $|K| \leqq\left|K_{12}\right|+\|A\|^{2}$. By (1.1) we have $K_{12}=O\left(1 / r^{3}\right)$ so (2.10) implies $\int_{S}\left|K_{12}\right|<\infty$. Thus we have

$$
\int_{S}|K|<\infty \text {. }
$$

We now use the function $f=\varphi$ in inequality (2.15) and let $\sigma \rightarrow \infty$ as above to conclude

$$
\int_{S}\left(R-K+\frac{1}{2}\|A\|^{2}\right) \leqq 0
$$

Since $R \geqq 0$, and $R>0$ outside a compact subset of $S$, we conclude

$$
\int_{S} K>0
$$

Remark 2.1. The Cohn-Vossen inequality says that $\int_{S} K \leqq 2 \pi \chi(S)$, where $\chi(S)$ is the Euler characteristic of $S$. Combining this with (2.18) we see immediately that $S$ is homeomorphic to $\mathbb{R}^{2}$.

In light of (2.18), the proof of Step 3 will be finished if we can show $\int_{S} K \leqq 0$. Since this is a very important part of our proof of Theorem 1, we give two proofs of this inequality. The first proof is conceptually very clear, and has the advantage of being more general than the second. The first proof, however, uses a deep theorem of R. Finn [11] and A. Huber [12] concerning the Gauss-Bonnet theorem on open Riemann surfaces, while the second uses no outside results and is special to our situation.

$$
\text { Claim } \int_{S} K \leqq 0 .
$$

First Proof. We first note that inequality (2.17) and Remark 2.1 imply, by a result of A. Huber [13], that $S$ is conformally equivalent to the complex plane. Thus there is a conformal diffeomorphism $F: \mathbb{C} \rightarrow S$. Let $D_{\sigma}$ denote the disk of radius $\sigma$ in $\mathbb{C}$, and let $C_{\sigma}$ be the circle of radius $\sigma$. For $i=1,2, \ldots$ let $L_{i}=$ length $\left(F\left(C_{i}\right)\right)$, and let $A_{i}=$ Area $\left(F\left(D_{i}\right)\right)$. The simply connected case of the theorem of R. Finn [11] and A. Huber [12] says that

$$
\int_{S} K=2 \pi-\lim _{i \rightarrow \infty} \frac{L_{i}^{2}}{2 A_{i}} .
$$

Thus to show $\int_{S} K \leqq 0$, it suffices to show

$$
\lim _{i \rightarrow \infty} \frac{L_{i}^{2}}{4 \pi A_{i}} \geqq 1 .
$$

Since $S$ is properly imbedded in $N$ with $S \cap\left(N \backslash N_{k}\right)$ compact, we see that $F\left(C_{i}\right)$ lies outside any compact subset of $N_{k}$ for $i$ sufficiently large. Thus for large $i$, we let $\tilde{L}_{i}$ 
be the Euclidean length of $F\left(C_{i}\right)$. Inequality (1.1) implies

$$
\tilde{L}_{i}^{2} \leqq(1+o(1)) L_{i}^{2} \quad \text { as } \quad i \rightarrow \infty .
$$

For the immersed disk $\Sigma_{i}$ of least Euclidean area with boundary curve $F\left(C_{i}\right)$, we have the well-known inequality whose proof can be found in [14]

$$
\tilde{A}\left(\Sigma_{i}\right) \leqq \frac{\tilde{L}_{i}^{2}}{4 \pi}
$$

where $\tilde{A}(\cdot)$ is Euclidean area. Let $\tilde{\Sigma}_{i}$ be an oriented surface of least Euclidean area among all surfaces of boundary $F\left(C_{i}\right)$ regardless of topological type (see Appendix). Since $\tilde{A}\left(\tilde{\Sigma}_{i}\right) \leqq \tilde{A}\left(\Sigma_{i}\right)$, we have

$$
\tilde{A}\left(\tilde{\Sigma}_{i}\right) \leqq \frac{\tilde{L}_{i}^{2}}{4 \pi} .
$$

Because $F\left(C_{i}\right)$ lies outside any compact set for $i$ sufficiently large, we can find a sequence $\sigma_{i} \rightarrow \infty$ with $\tilde{\Sigma}_{i} \cap B_{\sigma_{l}}(0) \subseteq \tilde{\Sigma}_{i} \backslash F\left(C_{i}\right)$. Since from Step 2 we know that $F\left(C_{i}\right)$ $\subseteq E_{h}=\left\{x \in \mathbb{R}^{3}:\left|x^{3}\right| \leqq h\right\}$, it follows that $\tilde{\Sigma}_{i} \subseteq E_{h}$ by the convex hull property of minimal surfaces. Since $\tilde{\Sigma}_{i}$ does not retract onto its boundary circle, there is a point $x_{0} \in \tilde{\Sigma}_{i} \cap\left\{\left(0,0, x^{3}\right): x^{3} \in \mathbb{R}\right\}$. A well-known inequality (see [15]) implies $\tilde{A}\left(\tilde{\Sigma}_{i} \cap B_{r}\left(x_{0}\right)\right) \geqq \pi r^{2}$. Thus we clearly have

$$
\tilde{A}\left(\tilde{\Sigma}_{i} \cap B_{\sigma_{i}}(0)\right) \geqq(1+o(1)) \pi \sigma_{i}^{2} .
$$

We wish to compare the $d s^{2}$-area of $\tilde{\Sigma}_{i}$ with the Euclidean area, but we cannot do it directly since $\tilde{\Sigma}_{i} \cap B_{\sigma_{0}}(0)$ may be nonempty, and $d s^{2}$ is not defined on this part of $\tilde{\Sigma}_{i}$. We get around this problem by modifying $\tilde{\Sigma}_{i}$ near 0 . Let $\bar{\sigma} \in\left[\sigma_{0}, \sigma_{0}+1\right]$ be such that $\partial B_{\bar{\sigma}}(0)$ and $\tilde{\Sigma}_{i}$ have transverse (or empty) intersection. We can then find a domain $\Omega_{i}$ on $\partial B_{\bar{\sigma}}(0)$ so that

$$
\partial \Omega_{i}=\tilde{\Sigma}_{i} \cap \partial B_{\bar{\sigma}}(0) .
$$

We then define a new surface $\hat{\Sigma}_{i}$ by

$$
\hat{\Sigma}_{i}=\left(\tilde{\Sigma}_{i} \backslash B_{\bar{\sigma}}(0)\right) \cup \Omega_{i} .
$$

Now (2.23) implies $\tilde{A}\left(\tilde{\Sigma}_{i}\right) \rightarrow \infty$, so we let $\tilde{A}_{i}=\tilde{A}\left(\hat{\Sigma}_{i}\right)$, and conclude

$$
\tilde{A}_{i} \leqq(1+o(1)) \tilde{A}\left(\tilde{\Sigma}_{i}\right)
$$

which combines with (2.22) to give

$$
\tilde{A}_{i} \leqq(1+o(1)) \frac{\tilde{L}_{i}^{2}}{4 \pi} .
$$

By the area minimizing property of $\tilde{\Sigma}_{i}$, we also have $\tilde{A}\left(\tilde{\Sigma}_{i}\right) \leqq \tilde{A}_{i}$, so $\tilde{A}_{i} \rightarrow \infty$. By choosing $\sigma_{i}$ smaller if necessary, we take

$$
A\left(\hat{\Sigma}_{i} \cap B_{\sigma_{i}}(0)\right) \leqq \sqrt{\tilde{A}_{i}}
$$

and $A\left(\hat{\Sigma}_{i} \cap B_{\sigma_{i}}(0)\right) \rightarrow \infty$. This can be done because of (2.23) and (1.1). If the $\sigma_{i}$ remain bounded, say $\sigma_{i} \leqq \varrho$ for all $i$, then by comparison as in the proof of (2.9) we would 
have

$$
\tilde{A}\left(\tilde{\Sigma}_{i} \cap B_{\varrho}(0)\right) \leqq 4 \pi \varrho^{2}
$$

which would imply that $A\left(\hat{\Sigma}_{i} \cap B_{\sigma_{i}}(0)\right)$ is a bounded sequence. Thus we must have $\sigma_{i} \rightarrow \infty$. It then follows by asymptotic flatness

$$
A\left(\hat{\Sigma}_{i} \backslash B_{\sigma_{t}}(0)\right) \leqq(1+o(1)) \tilde{A}_{i} .
$$

Combining this with (2.24a) we have

$$
A\left(\hat{\Sigma}_{i}\right) \leqq(1+o(1)) \tilde{A}_{i} \quad \text { as } \quad i \rightarrow \infty .
$$

Using the area minimizing property of $S$ and inequalities (2.21), (2.24), and (2.25) we have

$$
\begin{aligned}
A_{i} & \leqq A\left(\hat{\Sigma}_{i}\right) \leqq(1+o(1)) \tilde{A}_{i} \leqq(1+o(1)) \frac{\tilde{L}_{i}^{2}}{4 \pi} \\
& \leqq(1+o(1)) \frac{L_{i}^{2}}{4 \pi} \quad \text { as } \quad i \rightarrow \infty .
\end{aligned}
$$

We thus conclude $\lim _{i \rightarrow \infty} \frac{L_{i}^{2}}{4 \pi A_{i}} \geqq 1$ establishing (2.20). This completes the first proof of our claim.

Second Proof. We now give a proof of the claim in which we directly apply the Gauss-Bonnet theorem with boundary, and estimate the boundary terms. For any $x \in \mathbb{R}^{3}$, let $x^{\prime}=\left(x^{1}, x^{2}, 0\right)$, and let $r^{\prime}=\left|x^{\prime}\right|=\left(\left(x^{1}\right)^{2}+\left(x^{2}\right)^{2}\right)^{1 / 2}$. Consider the cylinder

$$
P_{\sigma}=\left\{x \in \mathbb{R}^{3}: r^{\prime} \leqq \sigma\right\} .
$$

For any $\sigma>\sigma_{0}$ for which $\partial P_{\sigma} \cap S$ is transverse, we have by Remark 2.1, at least one circle in this intersection which is not homologous to zero in $\mathbb{R}^{3} \backslash P_{\sigma_{0}}$. Choose one of these circles, and let $D_{\sigma}$ be the connected component of this circle in $S \cap\left[\left(N \backslash N_{k}\right) \cup P_{\sigma}\right]$. We claim that for $\sigma$ sufficiently large, $D_{\sigma}$ is a disk. To see this, recall from the first proof that $S$ is conformally equivalent to $\mathbb{C}$, so we have a conformal diffeomorphism $F: \mathbb{C} \rightarrow S$. Now $F^{-1}\left(D_{\sigma}\right)$ is a bounded, connected region in $\mathbb{C}$. By transversality, the function $r^{\prime}$ changes sign across each boundary component of $F^{-1}\left(D_{\sigma}\right)$. If $F^{-1}\left(D_{\sigma}\right)$ is not simply connected, then there is a bounded domain $\mathcal{O}$ contained in $\mathbb{C} \backslash F^{-1}\left(D_{\sigma}\right)$. Thus on $\partial F(\mathcal{O})$ we have $r^{\prime}=\sigma$, and inside $F(\mathcal{O})$ at some points we have $r^{\prime}>\sigma$. Thus $r^{\prime}$ takes a maximum at some point of $F(\mathcal{O})$. We claim that $\left(r^{\prime}\right)^{2}$ is a subharmonic function on $S$ for $r^{\prime}$ sufficiently large, which will give a contradiction. We calculate

$$
\Delta x^{i}=\sum_{j=1}^{2} e_{j} e_{j} x^{i}-\nabla_{e_{j}} e_{j} x^{i} .
$$

Now $e_{j} x^{i}=\left\langle e_{j}, \frac{\partial}{\partial x^{i}}\right\rangle+O(1 / r)$, so

$$
\begin{aligned}
\Delta x^{i} & =\sum_{j=1}^{2}\left[\left\langle\nabla_{e_{j}} e_{j}, \frac{\partial}{\partial x^{i}}\right\rangle+h_{j j}\left\langle V, \frac{\partial}{\partial x^{i}}\right\rangle-\left\langle\nabla_{e_{j}} e_{j}, \frac{\partial}{\partial x^{i}}\right\rangle\right]+O\left(1 / r^{2}\right) \\
& =O\left(1 / r^{2}\right)
\end{aligned}
$$


since $\sum_{j=1}^{2} h_{j j}=0$. Thus we have

$$
\Delta\left(r^{\prime}\right)^{2}=2 \sum_{i, j=1}^{2}\left\langle e_{i}, \frac{\partial}{\partial x^{j}}\right\rangle^{2}+O(1 / r)
$$

Since both $\left\{e_{1}, e_{2}\right\}$ and $\left\{\frac{\partial}{\partial x^{1}}, \frac{\partial}{\partial x^{2}}\right\}$ span 2-dimensional subspace of $\mathbb{R}^{3}$, they must intersect in at least a line, so the norm of their projection is asymptotically bounded below, i.e. $\sum_{i, j=1}^{2}\left\langle e_{i}, \frac{\partial}{\partial x^{j}}\right\rangle^{2} \geqq 1-O(1 / r)$. So we have

$$
\Delta\left(r^{\prime}\right)^{2} \geqq 2-O(1 / r) \quad \text { as } \quad r \rightarrow \infty \text {. }
$$

Thus for $r$ sufficiently large, in particular for $r^{\prime}$ sufficiently large we have $\Delta\left(r^{\prime}\right)^{2}>0$. Thus it follows that $D_{\sigma}$ is a disk for $\sigma$ large.

We may choose the $D_{\sigma}$ to be increasing, so that $D_{\bar{\sigma}} \supset D_{\sigma}$ when $\bar{\sigma}>\sigma$. Since $S$ is connected, the $D_{\sigma}$ form an exhaustion of $S$, and we apply the Gauss-Bonnet theorem on $D_{\sigma}$, so that

$$
\int_{D_{\sigma}} K=2 \pi-\int_{\partial D_{\sigma}} k
$$

where $k$ is the geodesic curvature of $\partial D_{\sigma}$ relative to the inner normal. Thus, our proof will be complete if we can find a sequence $\sigma_{i} \rightarrow \infty$ so that

$$
\int_{\partial D_{\sigma_{i}}} k \geqq 2 \pi-o(1) \text { as } i \rightarrow \infty \text {. }
$$

In a neighborhood of $\partial D_{\sigma}$, we choose a frame $e_{1}, e_{2}, e_{3}$ where $e_{1}$ the positively oriented unit tangent vector of $\partial D_{\sigma}, e_{2}$ is the inner normal to $D_{\sigma}$, and $e_{3}=v$ is the unit normal of $S$ in $\mathbb{R}^{3}$ relative to $d s^{2}$. The geodesic curvature $k$ is given by

$$
k=\left\langle D_{e_{1}} e_{1}, e_{2}\right\rangle \text {. }
$$

Since $r^{\prime}=\sigma$ on $\partial D_{\sigma}$, we have $\left\langle e_{1}, D r^{\prime}\right\rangle=0$ on $\partial D_{\sigma}$. Differentiating this with respect to $e_{1}$ gives

$$
\left\langle D_{e_{1}} e_{1}, D r^{\prime}\right\rangle+\left\langle e_{1}, D_{e_{1}} D r^{\prime}\right\rangle=0 .
$$

Now (1.1) implies that $D r^{\prime}=\frac{x^{\prime}}{\sigma}+O(1 / \sigma)$ and

$$
D_{e_{1}} D r^{\prime}=\frac{e_{1}}{\sigma}-\frac{1}{\sigma}\left\langle e_{1}, \frac{\partial}{\partial x^{3}}\right\rangle \frac{\partial}{\partial x^{3}}+O\left(1 / \sigma^{2}\right)
$$

so we have

$$
\left\langle D_{e_{1}} e_{1}, \frac{x^{\prime}}{\sigma}\right\rangle+1 / \sigma-1 / \sigma\left\langle e_{1}, \frac{\partial^{2}}{\partial x^{3}}\right\rangle^{2}=O(1 / \sigma)\left\|D_{e_{1}} e_{1}\right\|+O\left(1 / \sigma^{2}\right) .
$$

Since $D_{e_{1}} e_{1}=k e_{2}-h_{11} v$, this gives

$$
k\left\langle e_{e}, \frac{x^{\prime}}{\sigma}\right\rangle+1 / \sigma-h_{11}\left\langle v, \frac{x^{\prime}}{\sigma}\right\rangle-\frac{1}{\sigma}\left\langle e_{1}, \frac{\partial}{\partial x^{3}}\right\rangle^{2}=O(1 / \sigma)\left\|D_{e_{1}} e_{1}\right\|+O\left(1 / \sigma^{2}\right) .
$$


Suppose $\sigma \in[\bar{\sigma}, 2 \bar{\sigma}]$. We now apply the divergence theorem for the vector field $\frac{\partial}{\partial x^{3}}$ on the volume enclosed by $D_{2 \bar{\sigma}} \backslash D_{\bar{\sigma}}, \partial P_{2 \bar{\sigma}}, \partial P_{\bar{\sigma}}$, and the plane annulus $\Omega_{\bar{\sigma}}=\left\{x: x^{3}=-h, \bar{\sigma} \leqq r^{\prime} \leqq 2 \bar{\sigma}\right\}$ where $h$ is a bound on $\left|x^{3}\right|$ for $x \in S \cap N_{k}$ (see Step 2). By (1.1), $\operatorname{div} \frac{\partial}{\partial x^{3}}=O\left(1 / \bar{\sigma}^{2}\right)$, so we have

$$
\int_{D_{2 \bar{\sigma}} \backslash D_{\bar{\sigma}}}\left\langle v, \frac{\partial}{\partial x^{3}}\right\rangle-A\left(\Omega_{\sigma}\right)=O(1)
$$

where we have used the fact that $\left\langle\frac{\partial}{\partial x^{3}}, \mathbf{n}\right\rangle=O(1 / \sigma)$ where $\mathbf{n}$ is the unit normal of $\partial P_{2 \bar{\sigma}}$ and $\partial P_{\bar{\sigma}}$. Applying the area minimizing property of $S$ on $D_{2 \bar{\sigma}} \backslash D_{\bar{\sigma}}$ as compared with the union of $\Omega_{\bar{\sigma}}$ and the part of $\partial P_{2 \bar{\sigma}} \cup \partial P_{\bar{\sigma}}$ between $S$ and $\Omega_{\bar{\sigma}}$, we have

$$
A\left(D_{2 \bar{\sigma}} \backslash D_{\bar{\sigma}}\right) \leqq A\left(\Omega_{\bar{\sigma}}\right)+O(\bar{\sigma}) .
$$

Combined with the above inequality this gives

$$
\int_{D_{2 \bar{\sigma}} \backslash D_{\bar{\sigma}}} 1-\left\langle v, \frac{\partial}{\partial x^{3}}\right\rangle \leqq O(\bar{\sigma}) .
$$

The coarea formula (see [16, p. 258]) gives

$$
\int_{\bar{\sigma}}^{2 \bar{\sigma}} \int_{D_{2 \bar{\sigma}} \cap \partial P_{t}}\left(1-\left\langle v, \frac{\partial}{\partial x^{3}}\right\rangle\right) d s d t=\int_{D_{2 \bar{\sigma}} \cap\left(P_{2 \bar{\sigma}} \backslash P \overline{\bar{\sigma}}\right)}\left\|\nabla r^{\prime}\right\|\left(1-\left\langle v, \frac{\partial}{\partial x^{3}}\right\rangle\right)
$$

where $d s$ is arclength on $D_{2 \bar{\sigma}} \cap \partial P_{t}$. Since

$$
D_{2 \bar{\sigma}} \cap\left(P_{2 \bar{\sigma}} \backslash P_{\bar{\sigma}}\right) \cong D_{2 \bar{\sigma}} \backslash D_{\bar{\sigma}},
$$

we can combine these inequalities

$$
\int_{\bar{\sigma}}^{2 \bar{\sigma}} \int_{D_{2 \bar{\sigma}} \cap \partial P_{t}}\left(1-\left\langle v, \frac{\partial}{\partial x^{3}}\right\rangle\right) d s d t \leqq O(\bar{\sigma}) .
$$

Again using the coarea formula we have

$$
\int_{\bar{\sigma}}^{2 \bar{\sigma}} L\left(D_{2 \bar{\sigma}} \cap \partial P_{t}\right) d t=\int_{D_{2 \bar{\sigma}} \cap\left(P_{2 \bar{\sigma}} \backslash P_{\bar{\sigma}}\right)}\left\|\nabla r^{\prime}\right\| .
$$

Combined with (2.28) this gives

$$
\int_{\bar{\sigma}}^{2 \bar{\sigma}} L\left(D_{2 \bar{\sigma}} \cap \partial P_{t}\right) d t=O\left(\bar{\sigma}^{2}\right) .
$$

We must now bound the second fundamental form of $S$ on $\partial D_{\sigma}$. To do this, we apply inequality (2.16) with the following choice of $g$

$$
g(x)=\left\{\begin{array}{ccc}
0 & \text { for } & x \in S \cap\left[\left(N \backslash N_{k}\right) \cup P_{\sqrt{\bar{\sigma}}}\right] \\
\log \frac{r^{\prime}}{\sqrt{\bar{\sigma}}} & \text { for } & x \in S \cap\left(P_{\bar{\sigma}} \backslash P_{\sqrt{\bar{\sigma}}}\right) \\
\frac{\log \sqrt{\bar{\sigma}}}{1} & \text { for } \quad x \notin S \cap\left[\left(N \backslash N_{k}\right) \cup P_{\bar{\sigma}}\right] .
\end{array}\right.
$$


This implies

$$
\int_{S \backslash\left[\left(N \backslash N_{k}\right) \cup P_{\bar{\sigma}}\right]}\|A\|^{2} \leqq \frac{2}{(\log \sqrt{\bar{\sigma}})^{2}} \int_{S \cap(P \overline{\bar{\sigma}} \backslash P \sqrt{\bar{\sigma}})} \frac{\left\|\nabla r^{\prime}\right\|^{2}}{\left(r^{\prime}\right)^{2}}+\int_{S \backslash\left[N \backslash N_{k}\right) \cup P_{\sqrt{\bar{\sigma}}]}}|\operatorname{Ric}(v)| .
$$

This implies by (1.1)

$$
\int_{D_{2 \bar{\sigma}} \cap\left(P_{2 \bar{\sigma}} \backslash P_{\bar{\sigma}}\right)}\|A\|^{2} \leqq \frac{2 C}{(\log \sqrt{\bar{\sigma}})^{2}} \int_{S \cap\left(P_{\left.\bar{\sigma} \backslash P_{\bar{\sigma}}\right)}\right.} \frac{1}{\left(r^{\prime}\right)^{2}}+\int_{S \backslash\left(\left(N \backslash N_{k}\right) \cup P_{\sqrt{\sigma}}\right]} O\left(1 / r^{3}\right) .
$$

Similar reasoning as that used in deriving (2.10) and (2.11) using $r^{\prime}$ in place of $r$ and the fact that $x^{3}$ is bounded on $S \cap N_{k}$ implies

$$
\begin{gathered}
\int_{S \cap\left(P \overline{\bar{\sigma}} \backslash P_{\sqrt{\bar{\sigma}})}\right.} \frac{1}{\left(r^{\prime}\right)^{2}}=O(\log \sqrt{\bar{\sigma}}), \\
\int_{S \backslash\left[\left(N \backslash N_{k}\right) \cup P_{\sqrt{\bar{\sigma}}]}\right.} O\left(1 / r^{3}\right)=O\left(1 / \bar{\sigma}^{1 / 2}\right) .
\end{gathered}
$$

It therefore follows that

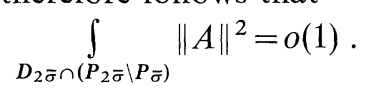

The coarea formula gives

$$
\int_{\bar{\sigma}}^{2 \bar{\sigma}} \int_{D_{2 \bar{\sigma}} \cap \partial P_{t}}\|A\|^{2} d t=\int_{D_{2 \bar{\sigma}} \cap\left(P_{2 \bar{\sigma}} \backslash P_{\bar{\sigma}}\right)}\|A\|^{2}\left\|\nabla r^{\prime}\right\|
$$

This then implies

$$
\int_{\bar{\sigma}}^{2 \bar{\sigma}} \int_{D_{2 \bar{\sigma} \cap \partial P_{t}}}\|A\|^{2} d t=o(1)
$$

Now (2.29), (2.30), and (2.31) imply that there exists $\sigma \in[\bar{\sigma}, 2 \bar{\sigma}]$ satisfying

$$
\begin{aligned}
& \int_{D_{2 \bar{\sigma} \cap \partial P_{\sigma}}}\left(1-\left\langle v, \frac{\partial}{\partial x^{3}}\right\rangle\right) \leqq O(1), \quad L\left(D_{2 \bar{\sigma}} \cap \partial P_{\sigma}\right)=O(\sigma), \\
& \int_{D_{2 \bar{\sigma} \cap \partial P_{\sigma}}}\|A\|^{2}=o(1 / \sigma) \text { as } \sigma \rightarrow \infty .
\end{aligned}
$$

Applying the Schwarz inequality and the condition on boundary length we have

$$
\left(\int_{D_{2 \bar{\sigma}} \cap \partial P_{\sigma}}\|A\|\right)^{2} \leqq L\left(D_{2 \bar{\sigma}} \cap \partial P_{\sigma}\right) \cdot o(1 / \sigma)=o(1) .
$$

Since $\partial D_{\sigma}$ is one component of $D_{2 \bar{\sigma}} \cap \partial P_{\sigma}$, we have shown

$$
\begin{gathered}
\int_{\partial D_{\sigma}} 1-\left\langle v, \frac{\partial}{\partial x^{3}}\right\rangle \leqq O(1) \\
L\left(\partial D_{\sigma}\right)=O(\sigma) \\
\left.\int_{\partial D_{\sigma}}\|A\|=O(1)\right) .
\end{gathered}
$$


We use (2.32) to estimate the terms in (2.27). Note that (2.27) and (2.32) imply

$$
\int_{\partial D_{\sigma}}\left|k\left\langle e_{2}, \frac{x^{\prime}}{\sigma}\right\rangle\right|=O(1)+O(1 / \sigma) \int_{\partial D_{\sigma}}\left\|D_{e_{1}} e_{1}\right\|+\frac{1}{\sigma} \int_{\partial D_{\sigma}}\left\langle e_{1}, \frac{\partial}{\partial x^{3}}\right\rangle^{2} .
$$

To bound the last term, we note that by (1.1) we have

$$
\left\langle e_{1}, \frac{\partial}{\partial x^{3}}\right\rangle^{2}+\left\langle e_{2}, \frac{\partial}{\partial x^{3}}\right\rangle^{2}=1-\left\langle v, \frac{\partial}{\partial x^{3}}\right\rangle^{2}+O(1 / \sigma)
$$

which implies by (2.32) that

$$
\int_{\partial D_{\sigma}}\left\langle e_{1}, \frac{\partial}{\partial x^{3}}\right\rangle^{2}+\left\langle e_{2}, \frac{\partial}{\partial x^{3}}\right\rangle^{2}=O(1) \text {. }
$$

We now give a pointwise lower bound on $\left|\left\langle e_{2}, \frac{\partial}{\partial x^{3}}\right\rangle\right|$. In fact we show that

$$
\sup _{\partial D_{\sigma}}\left(1+\left\langle\frac{x^{\prime}}{\sigma}, e_{2}\right\rangle\right)=o(1) \text {. }
$$

We first note that by (1.1)

$$
\begin{aligned}
1-\left\langle\frac{x^{\prime}}{\sigma}, e_{2}\right\rangle^{2} & =\left\langle\frac{x^{\prime}}{\sigma}, v\right\rangle^{2}+O(1 / \sigma)=\left\langle\frac{x^{\prime}}{\sigma}, v-\frac{\partial}{\partial x^{3}}\right\rangle^{2}+O(1 / \sigma) \\
& \leqq\left\|v-\frac{\partial}{\partial x^{3}}\right\|^{2}+O(1 / \sigma) \\
& =2\left(1-\left\langle v, \frac{\partial}{\partial x^{3}}\right\rangle\right)+O(1 / \sigma)
\end{aligned}
$$

Then applying (2.32) gives

$$
\int_{\partial D_{\sigma}} 1-\left\langle\frac{x^{\prime}}{\sigma}, e_{2}\right\rangle^{2}=O(1) \text {. }
$$

Now since $e_{2}$ is the inner normal to $D_{\sigma}$, and $D r^{\prime}=\frac{x^{\prime}}{\sigma}+O(1 / \sigma)$ is the outer normal, we have $\left\langle\frac{x^{\prime}}{\sigma}, e_{2}\right\rangle \leqq O(1 / \sigma)$, so

$$
\begin{aligned}
1-\left\langle\frac{x^{\prime}}{\sigma}, e_{2}\right\rangle^{2} & =\left(1-\left\langle\frac{x^{\prime}}{\sigma}, e_{2}\right\rangle\right)\left(1+\left\langle\frac{x^{\prime}}{\sigma}, e_{2}\right\rangle\right) \\
& \geqq(1-O(1 / \sigma))\left(1+\left\langle\frac{x^{\prime}}{\sigma}, e_{2}\right\rangle\right)+O(1 / \sigma) .
\end{aligned}
$$

Combining with (2.36) then gives

$$
\int 1+\left\langle\frac{x^{\prime}}{-}, e_{\eta}\right\rangle=O(1) .
$$


Since $\partial D_{\sigma}$ is not homologous to zero in $\mathbb{R}^{2} \backslash P_{\sigma_{0}}$, its projection onto the $x^{1} x^{2}$-plane must be the circle of radius $\sigma$ centered at zero. We therefore have

$$
L\left(\partial D_{\sigma}\right) \geqq 2 \pi \sigma-O(1) .
$$

Now (2.37) and (2.38) together imply that there is a point $x_{0} \in \partial D_{\sigma}$ with

$$
1+\left\langle\frac{x^{\prime}}{\sigma}, e_{2}\right\rangle\left(x_{0}\right)=O(1 / \sigma) \text {. }
$$

Differentiating $1+\left\langle\frac{x^{\prime}}{\sigma}, e_{2}\right\rangle$ along $\partial D_{\sigma}$ gives

$$
\begin{aligned}
e_{1}\left[1+\left\langle\frac{x^{\prime}}{\sigma}, e_{2}\right\rangle\right]= & \frac{1}{\sigma}\left\langle e_{1}, e_{2}\right\rangle-\frac{1}{\sigma}\left\langle\frac{\partial}{\partial x^{3}}, e_{1}\right\rangle\left\langle\frac{\partial}{\partial x^{3}}, e_{2}\right\rangle \\
& -h_{12}\left\langle\frac{x^{\prime}}{\sigma}, v\right\rangle+O(1 / \sigma)^{2} \\
\leqq & \frac{1}{2 \sigma}\left(\left\langle\frac{\partial}{\partial x^{3}}, e_{1}\right\rangle^{2}+\left\langle\frac{\partial}{\partial x^{3}}, e_{2}\right\rangle^{2}\right)+\|A\|+O\left(1 / \sigma^{2}\right) .
\end{aligned}
$$

Applying (2.32) and (2.34) we see

$$
\int_{\partial D_{\sigma}}\left|e_{1}\left[1+\left\langle\frac{x^{\prime}}{\sigma}, e_{2}\right\rangle\right]\right|=o(1) \text {. }
$$

We now write for any $x \in \partial D_{\sigma}$,

$$
1+\left\langle\frac{x^{\prime}}{\sigma}, e_{e}\right\rangle(x)=1+\left\langle\frac{x^{\prime}}{\sigma}, e_{2}\right\rangle\left(x_{0}\right)+\int_{x_{0}}^{x} e_{1}\left[1+\left\langle\frac{x^{\prime}}{\sigma}, e_{2}\right\rangle\right] .
$$

Thus combining this with (2.39) and (2.40) we have established (2.35).

Now (2.33), (2.34) and (2.35) together imply

$$
\int_{\partial D_{\sigma}}|k|=O(1)+O(1 / \sigma) \int_{\partial D_{\sigma}}\left\|D_{e_{1}} e_{1}\right\| \text {. }
$$

Since $D_{e_{1}} e_{1}=k e_{2}-h_{11} v$ we have

$$
\left\|D_{e_{1}} e_{1}\right\| \leqq|k|+\|A\| \text {, }
$$

so integrating and applying (2.32) we have

$$
\int_{\partial D_{\sigma}}\left\|D_{e_{1}} e_{1}\right\| \leqq \int_{\partial D_{\sigma}}|k|+o(1)
$$

Combining this with (2.41) gives

$$
\begin{gathered}
\int_{\partial D_{\sigma}}\left\|D_{e_{1}} e_{1}\right\|=O(1) \\
\int_{\partial D_{\sigma}^{\prime}}|k|=O(1) .
\end{gathered}
$$


We may now rewrite (2.27) using (2.32), (2.34), and (2.42)

$$
\int_{\partial D_{\sigma}} k \geqq \int_{\partial D_{\sigma}} k\left(1+\left\langle\frac{x^{\prime}}{\sigma}, e_{2}\right\rangle\right)+1 / \sigma L\left(\partial D_{\sigma}\right)-o(1) .
$$

Applying (2.35), (2.38), and (2.42) then gives

$$
\int_{\partial D_{\sigma}} k \geqq 2 \pi-o(1) \text {. }
$$

Since this holds for $\sigma$ arbitrarily large (in any interval $[\bar{\sigma}, 2 \bar{\sigma}], \bar{\sigma}$ sufficiently large), we can choose a sequence $\sigma_{i} \rightarrow \infty$ for which (2.26) holds. This completes the second proof of our claim.

This finishes the proof of Theorem 1 .

\section{Proof of Theorem 2}

In this section we prove Theorem 2 which says that if for some end $N_{k},(1.2)$ holds, the total mass of $M_{k}$ is zero, and $R \geqq 0$, then $d s^{2}$ is flat. We first note that by throwing away the other ends outside a convex ball, we may assume that $N$ has only one end, so that $N \backslash N_{k}$ is compact.

We will need the following Sobolev inequality for functions with compact support on $N$.

Lemma 3.1. There is a constant $c_{1}>0$ depending on $N$ and the constants $k_{1}, k_{2}, k_{3}$ of (1.1) so that for any function $\zeta$ with compact support on $N$, we have the inequality

$$
\left(\int_{N} \zeta^{6}\right)^{1 / 3} \leqq c_{1} \int_{N}\|D \zeta\|^{2}
$$

Note that we do not require $\zeta=0$ on $\partial N$.

Proof. We prove the inequality by contradiction. If it were not true, we could find a sequence of functions $f_{i}$ with compact support and with

$$
\int_{N} f_{i}^{6}=1, \quad \int_{N}\left\|D f_{i}\right\|^{2} \leqq 1 / i
$$

Since $N_{k}$ is identified with $\mathbb{R}^{3} \backslash B_{\sigma_{0}}(0)$ and $d s^{2}$ is uniformly equivalent to the Euclidean metric, we have the inequality which follows from the Euclidean inequality (see proof in $[17$, p. 80-81]).

$$
\left(\int_{N_{k}}\left(f_{i}\right)^{6}\right)^{1 / 3} \leqq(\text { const }) \int_{N_{k}}\left\|D f_{i}\right\|^{2}
$$

Thus by (3.1) we have $\int_{N_{k}} f_{i}^{6} \rightarrow 0$, so we have $f_{i} \rightarrow 0$ in $L^{6}$-norm on $N_{k}$. If we choose a precompact coordinate neighborhood $\mathcal{O} \subseteq N$, for any $C^{1}$ function $g$ defined on $\mathcal{O}$ we have the following inequality which comes directly from the Euclidean inequality of the same form

$$
\inf _{\beta \in \mathbb{R}}\left(\int_{\mathscr{O}}(g-\beta)^{6}\right)^{1 / 3} \leqq(\text { const }) \int_{\mathscr{O}}\|D g\|^{2} .
$$


Applying this inequality and (3.1) to the functions $\left.f_{i}\right|_{\mathcal{O}}$, we find a sequence $\beta_{i}$ so that $\int_{\mathcal{O}}\left(f_{i}-\beta_{i}\right)^{6} \rightarrow 0$

Since $\int_{N} f_{i}^{6}=1$, the sequence $\beta_{i}$ is a bounded sequence, and by extracting a subsequence we may assume $\beta_{i} \rightarrow \beta$. Thus we have $f_{i} \rightarrow \beta$ in $L^{6}$-norm on $\mathcal{O}$. Since $f_{i} \rightarrow 0$ in $L^{6}$-norm on $N_{k}$, we must have $\beta=0$ on each coordinate neighborhood $\mathcal{O}$, so we have $f_{i} \rightarrow 0$ in $L^{6}$-norm on $N$ in contradiction to (3.1). This proves Lemma 3.1 .

We will have need to study equations of the form

$$
\Delta v-f v=h \text { on } N
$$

where $f, h$ are functions which satisfy

$$
\begin{aligned}
& |f| \leqq k_{7}\left(1+r^{+5}\right)^{-1}, \quad|h| \leqq k_{7}\left(1+r^{5}\right)^{-1}, \\
& |\partial f| \leqq k_{8}\left(1+r^{5}\right)^{-1}, \quad|\partial h| \leqq k_{8}\left(1+r^{5}\right)^{-1}
\end{aligned}
$$

on $N_{k}$. Let $f_{+}, f_{-}$be the positive and negative parts of $f$, so that $f=f_{+}-f_{-}$and $|f|=f_{+}+f_{-}$.

Lemma 3.2. Suppose (1.1) holds and $N_{k}$ has zero total mass. There is a number $\varepsilon_{0}>0$ depending only on $N$ and $k_{1}, k_{2}, k_{3}$ of (1.1) so that if

$$
\left(\int_{N}\left(f_{-}\right)^{3 / 2}\right)^{2 / 3} \leqq \varepsilon_{0}
$$

then (3.2) has a unique solution $v$ defined on $N$ satisfying $v=O(1 / r)$ as $r \rightarrow \infty$ and $\frac{\partial v}{\partial \mathbf{n}}=0$ on $\partial N$, where $\mathbf{n}$ is the outward unit normal vector to $\partial N$. Moreover, the solution $v$ has the properties

$$
\begin{gathered}
v=\frac{A}{r}+\omega, \quad|\omega| \leqq k_{9}\left(1+r^{2}\right)^{-1} \\
|\partial \omega| \leqq k_{10}\left(1+r^{3}\right)^{-1}, \quad|\partial \partial \omega| \leqq k_{11}\left(1+r^{4}\right)^{-1}
\end{gathered}
$$

on $N_{k}$, where $A=-\frac{1}{4 \pi} \int_{N} f v+h$, and the constants $k_{9}, k_{10}, k_{11}$ depend only on $k_{1}, k_{2}$, $k_{3}$.

Proof. Throughout the proof we use $c_{1}, c_{2}, c_{3} \ldots$ to denote constants depending only on $k_{1}, k_{2}, k_{3}$.

To prove the existence of $v$ we solve the problem for $\sigma>\sigma_{0}$

$$
\left\{\begin{array}{cl}
\Delta v_{\sigma}-f v_{\sigma}=h & \text { on } \quad N^{\sigma}=\left(N \backslash N_{k}\right) \cup\left(B_{\sigma}(0) \cap N_{k}\right) \\
v_{\sigma}=0 & \text { on } \quad \partial B_{\sigma}(0) \\
\frac{\partial v_{\sigma}}{\partial \mathbf{n}}=0 & \text { on } \quad \partial N .
\end{array}\right.
$$


If $v_{\sigma}$ satisfies (3.4), we can multiply by $v_{\sigma}$ and integrate by parts to obtain an integral bound on $v_{\sigma}$ as follows

$$
\begin{aligned}
\int_{N^{\sigma}}\left\|D v_{\sigma}\right\|^{2} & =-\int_{N^{\sigma}} f v_{\sigma}^{2}-\int_{N^{\sigma}} h v_{\sigma} \\
& \leqq \int_{N^{\sigma}}\left(f_{-}\right) v_{\sigma}^{2}+\int_{N^{\sigma}}|h|\left|v_{\sigma}\right| \\
& \leqq\left(\int_{N^{\sigma}} f_{-}^{3 / 2}\right)^{2 / 3}\left(\int_{N^{\sigma}} v_{\sigma}^{6}\right)^{1 / 3}+\left(\int_{N^{\sigma}}|h|^{6 / 5}\right)^{5 / 6}\left(\int_{N^{\sigma}} v_{\sigma}^{6}\right)^{1 / 6} .
\end{aligned}
$$

We note that if $h=0$, we can apply Lemma (3.1) to obtain

$$
\int_{N^{\sigma}}\|D v\|^{2} \leqq \varepsilon_{0} c_{1} \int_{N^{\sigma}}\left\|D v_{\sigma}\right\|^{2} .
$$

Thus if we choose $\varepsilon_{0}<1 / c_{1}$, we see that $\Delta v-f v$ has trivial kernel for problem (3.4), and hence standard linear elliptic theory (see [18, p. 262]) implies the existence of a unique smooth solution $v_{\sigma}$ of (3.4). By (3.3) we have $\left(\int_{N^{\sigma}}|h|^{6 / 5}\right)^{5 / 6} \leqq c_{2}$, a constant independent of $\sigma$. Applying this, Lemma 3.1, and the hypotheses we have

$$
\left(\int_{N^{\sigma}} v_{\sigma}^{6}\right)^{1 / 3} \leqq \varepsilon_{0} c_{1}\left(\int_{N^{\sigma}} v_{\sigma}^{6}\right)^{1 / 3}+c_{1} c_{2}\left(\int_{N^{\sigma}} v_{\sigma}^{6}\right)^{1 / 6}
$$

Choosing $\varepsilon_{0}=\frac{1}{3 c_{1}}$, and using the inequality $|a b| \leqq \frac{1}{3} a^{2}+\frac{3}{4} b^{2}$ we have

$$
\left(\int_{N^{\sigma}} v_{\sigma}^{6}\right)^{1 / 3} \leqq \frac{1}{3}\left(\int_{N^{\sigma}} v_{\sigma}^{6}\right)^{1 / 3}+\frac{1}{3}\left(\int_{N^{\sigma}} v_{\sigma}^{6}\right)^{1 / 3}+\frac{3}{4}\left(c_{1} c_{2}\right)^{2}
$$

which gives

$$
\int_{N^{\sigma}} v_{\sigma}^{6} \leqq c_{3}
$$

where $c_{3}=\left(\frac{9}{4}\left(c_{1} c_{2}\right)^{2}\right)^{3}$. Standard linear elliptic estimates (see [17, p. 161] for the interior estimate and $[17$, p. 242] for the estimate on $\partial N$ ) now imply that $\left\{v_{\sigma}: \sigma>\sigma_{0}\right\}$ is equicontinuous in $C^{2}$ topology on compact subsets of $N$. Thus we may choose a sequence $\sigma_{i} \rightarrow \infty$ so that $v_{\sigma_{l}} \rightarrow v$ uniformly in $C^{2}$-norm on compact subsets of $N$. Thus $v$ is a solution of (3.2) defined on $N$ satisfying

$$
\begin{aligned}
& \frac{\partial v}{\partial \mathbf{n}}=0 \quad \text { on } \quad \partial N, \quad \text { and } \\
& \int_{N} v^{6} \leqq c_{3}, \quad \sup _{N}|v| \leqq c_{4} .
\end{aligned}
$$

(The supremum estimate follows from the $L^{6}$ estimate and standard linear theory [17, p. 161].) 
To analyze the asymptotic behavior of $v$, we derive a potential theoretic expression for $v$. For $x, y \in N_{k}$ let

$$
\varrho_{x}(y)=\left[\sum_{i, j=1}^{3} g_{i j}(x)\left(y^{i}-x^{i}\right)\left(y^{j}-x^{j}\right)\right]^{1 / 2} .
$$

By direct calculation we have

$$
\Delta_{y}\left[\varrho_{x}(y)\right]^{-1}=-4 \pi \delta_{x}(y)+\psi_{x}(y)
$$

where $\delta_{x}(y)$ is a point mass at $x$, and by $(1.1) \psi_{x}(y)$ satisfies

$$
\left|\psi_{x}(y)\right| \leqq c_{5}|x-y|^{-2}|x|^{-3} \quad \text { for } \quad y \in B_{1}(x) \text {. }
$$

Again by (1.1) we see that for $y \notin B_{1}(x)$ we have

$$
\left|\psi_{x}(y)\right| \leqq c_{6}\left[\frac{1}{(1+|y|)^{2}|x-y|^{3}}+\frac{1}{|x|^{2}|x-y|^{3}}+\frac{1}{(1+|y|)^{3}|x-y|^{2}}\right]
$$

Also we have

$$
\begin{aligned}
& c_{7}^{-1}|x-y| \leqq \varrho_{x}(y) \leqq c_{7}|x-y|, \quad c_{8}^{-1} \leqq\left|\partial_{y} \varrho_{x}(y)\right| \leqq c_{8}, \\
& \lim _{|x| \rightarrow \infty}|x|\left[\varrho_{x}(y)\right]^{-1}=1
\end{aligned}
$$

Multiplying (3.2) by $\left[\varrho_{x}(y)\right]^{-1}$, integrating by parts twice on the set $D_{\bar{\sigma}}(x)$ $=\left\{y \in N_{k}: \varrho_{x}(y) \leqq \bar{\sigma}\right\}$ where $\bar{\sigma} \in(\sigma / 2, \sigma)$ and $\sigma / 2 \gg|x|$, and applying (3.6)

$$
\begin{aligned}
4 \pi v(x)= & \int_{D \bar{\sigma}(x)} \psi_{x}(y) v(y) \sqrt{g(y)} d y-\int_{D \bar{\sigma}(x)}(f v+h)(y)\left[\varrho_{x}(y)\right]^{-1} \sqrt{g(y)} d y \\
& +\frac{1}{\bar{\sigma}} \int_{\left\{\varrho_{x}(y)=\bar{\sigma}\right\}} \frac{\partial v}{\partial \mathbf{n}} d A(y)-\int_{\left\{\varrho_{x}(y)=\bar{\sigma}\right\}} v(y) \frac{\partial}{\partial \mathbf{n}}\left[\varrho_{x}(y)\right]^{-1} d A(y) \\
& -\int_{\partial B_{\sigma_{0}}(0)} \frac{\partial v}{\partial \mathbf{n}}(y)\left[\varrho_{x}(y)\right]^{-1} d A(y)+\int_{\partial B_{\sigma_{0}}(0)} v(y) \frac{\partial}{\partial \mathbf{n}}\left[\varrho_{x}(y)\right]^{-1} d A(y)
\end{aligned}
$$

where $\sqrt{g} d y$ is the volume element of $d s^{2}$ and $d A$ is surface area with respect to $d s^{2}$. Applying Stokes' theorem we see from (3.2)

$$
\begin{aligned}
& \int_{\left\{\varrho_{x}(y)=\bar{\sigma}\right\}} \frac{\partial v}{\partial \mathbf{n}}(y) d A(y) \\
& =\int_{\partial B_{\sigma_{0}}(0)} \frac{\partial v}{\partial \mathbf{n}}+\int_{D \bar{\sigma}(x)} \Delta v=\int_{\partial B_{\sigma_{0}}(0)} \frac{\partial v}{\partial \mathbf{n}}+\int_{D \bar{\sigma}(x)}(f v+h) .
\end{aligned}
$$

From (3.3) and (3.5) we have

$$
\left|\int_{D_{\bar{\sigma}}(x)} f v+h\right| \leqq c_{9} .
$$


Therefore,

$$
\left|\int_{\left\{\varrho_{x}(y)=\bar{\sigma}\right\}} \frac{\partial v}{\partial \mathbf{n}}\right| \leqq\left|\int_{\partial B_{\sigma_{0}}(0)} \frac{\partial v}{\partial \mathbf{n}}\right|+c_{10} \leqq c_{11} .
$$

From (3.8), Area $\left\{\varrho_{x}(y)=\bar{\sigma}\right\} \leqq c_{12} \bar{\sigma}^{2}$, so we may apply (3.8)

$$
\begin{aligned}
\int_{\left\{\varrho_{x}(y)=\bar{\sigma}\right\}} v \frac{\partial}{\partial \mathbf{n}}\left[\varrho_{x}(y)\right]^{-1} \mid & \leqq c_{13} \bar{\sigma}^{-2} \int_{\left\{\varrho_{x}(y)=\bar{\sigma}\right\}}|v| \\
& \leqq c_{13} \bar{\sigma}^{-2}\left(c_{12} \bar{\sigma}^{2}\right)^{5 / 6}\left(\int_{\left\{d_{x}(y)=\bar{\sigma}\right\}} v^{6}\right)^{1 / 6} \\
& \leqq c_{14} \sigma^{-1 / 3}\left(\int_{\left\{\varrho_{x}(y)=\bar{\sigma}\right\}} v^{6}\right)^{1 / 6} .
\end{aligned}
$$

By (3.5) and the coarea formula we may choose $\bar{\sigma} \in(\sigma / 2, \sigma)$ so that

$$
\int_{\left\{\varrho_{x}(y)=\bar{\sigma}\right\}} v^{6} \leqq c_{15} \sigma^{-1} .
$$

Thus we have

$$
\left|\int_{\left\{\varrho_{x}(y)=\bar{\sigma}\right\}} v \frac{\partial}{\partial \mathbf{n}}\left[\varrho_{x}(y)\right]^{-1}\right| \leqq c_{16} \sigma^{-1 / 2} .
$$

We now let $\sigma \rightarrow \infty$ and apply (3.10) and (3.11) in (3.9) to get

$$
\begin{aligned}
4 \pi v(x)= & \int_{N_{k}} \psi_{x}(y) v(y) \sqrt{g(y)} d y \\
& -\int_{N_{k}}(f v+h)(y)\left[\varrho_{x}(y)\right]^{-1} \sqrt{g(y)} d y \\
& -\int_{\partial \boldsymbol{B}_{\sigma_{0}}(0)} \frac{\partial v}{\partial \mathbf{n}}\left[\varrho_{x}(y)\right]^{-1} d A(y) \\
& +\int_{\partial \boldsymbol{B}_{\sigma_{0}}(0)} v \frac{\partial}{\partial \mathbf{n}}\left[\varrho_{x}(y)\right]^{-1} d A(y) .
\end{aligned}
$$

From (3.5), (3.6), and (3.7) we have

$$
\begin{aligned}
\left.\mid \int_{N_{k}} \psi_{x}(y) v(y) \sqrt{g(y)}\right) d y \mid & \leqq \\
\leqq & c_{17}\left[\int_{B_{1}(x)}\left|\psi_{x}(y)\right| d y+\int_{N_{k} \backslash B_{1}(x)}\left|\psi_{x}(y)\right||v(y)| d y\right] \\
\leqq & c_{18}|x|^{-3}+c_{19}\left(\int _ { \mathbb { R } ^ { 3 } \backslash B _ { 1 } ( x ) } \left(\frac{1}{(1+|y|)^{2}|x-y|^{3}}+\frac{1}{(1+|y|)^{3}|x-y|^{2}}\right.\right. \\
& \left.\left.+\frac{1}{|x|^{2}|x-y|^{3}}\right)^{6 / 5} d y\right)^{5 / 6} .
\end{aligned}
$$


The following inequalities are easily checked

$$
\begin{aligned}
& \left(\int_{\mathbb{R}^{3} \backslash B_{1}(x)} \frac{d y}{(1+|y|)^{12 / 5}|x-y|^{18 / 5}}\right)^{5 / 6} \\
& \leqq\left(\int_{B_{\frac{|x|}{2}}(x) \backslash B_{1}(x)} \frac{d y}{(1+|y|)^{12 / 5}|x-y|^{18 / 5}}\right)^{5 / 6} \\
& +\left(\int_{B_{\frac{|x|}{2}}(0)} \frac{d y}{(1+|y|)^{12 / 5}|x-y|^{18 / 5}}\right)^{5 / 6} \\
& +\left(\int_{\mathbb{R}^{3} \backslash\left(B_{\frac{|x|}{2}}(0) \cup B_{\frac{|x|}{2}}(x)\right)} \frac{d y}{(1+|y|)^{12 / 5}|x-y|^{18 / 5}}\right)^{5 / 6} \\
& \leqq c_{20}\left(|x|^{-2}+|x|^{-5 / 2}+|x|^{-5 / 2}\right) \leqq c|x|^{-2} .
\end{aligned}
$$

Similarly

$$
\begin{aligned}
& \left(\int_{\mathbb{R}^{3} \backslash B_{1}(x)} \frac{d y}{(1+|y|)^{18 / 5}|x-y|^{12 / 5}}\right)^{5 / 6} \leqq c_{21}|x|^{-2} \\
& \int_{\mathbb{R}^{3} \backslash B_{1}(x)} \frac{d y}{|x-y|^{18 / 5}} \leqq c_{22} .
\end{aligned}
$$

Thus it follows from (3.13) that

$$
\left|\int_{N_{k}} \psi_{x}(y) v(y) \sqrt{g(y)} d y\right| \leqq c_{23}|x|^{-2} .
$$

It follows from (3.3), (3.5), and (3.8) that

$$
\begin{aligned}
& \left|\int_{\frac{|x|}{2}(x)}(f v+h)(y)\left[\varrho_{x}(y)\right]^{-1} \sqrt{g(y)} d y\right| \leqq c_{24}|x|^{-3} \\
& |x|\left[\varrho_{x}(y)\right]^{-1} \leqq c_{25} \text { for } y \notin \frac{B_{|x|}}{2}(x) .
\end{aligned}
$$

We may thus apply (3.8), (3.3) and the dominated convergence theorem to the functions $(f v+h)(y)|x|\left[\varrho_{x}(y)\right]^{-1} \sqrt{g(y)} \chi_{N_{k} \backslash B_{\frac{|x|}{2}}(x)}$ where $\chi_{A}$ denotes the characteristic function of $A$ to conclude that

$$
\begin{gathered}
\lim _{x \rightarrow \infty}|x| \int_{N_{k}}(f v+h)(y)\left[\varrho_{x}(y)\right]^{-1} \sqrt{g(y)} d y \\
=\int_{N_{k}}(f v+h)(y) \sqrt{g(y)} d y .
\end{gathered}
$$

Then by (3.12), (3.14), (3.8), and (3.15) we have

$$
\begin{aligned}
A= & \lim _{|x| \rightarrow \infty} 4 \pi|x| v(x)=-\int_{N_{k}}(f v+h)(y) \sqrt{g} d y \\
& -\int_{\partial B_{\sigma_{0}}(0)} \frac{\partial v}{\partial \mathbf{n}}
\end{aligned}
$$


so we may write

$$
v=\frac{A}{r}+\omega
$$

where

$$
\begin{aligned}
4 \pi \omega(x)= & \int_{N_{k}} \psi_{x}(y) v(y) \sqrt{g(y)} d y-\int_{N_{k}}(f v+h)(y)\left[\varrho_{x}(y)^{-1}-|x|^{-1}\right] \sqrt{g} d y \\
& -\int_{\partial B_{\sigma_{0}}(0)} \frac{\partial v}{\partial \mathbf{n}}\left[\varrho_{x}(y)^{-1}-|x|^{-1}\right] d A(y)+\int_{\partial B_{\sigma_{0}}(0)} v \frac{\partial}{\partial \mathbf{n}}\left[\varrho_{x}(y)\right]^{-1} d A(y) .
\end{aligned}
$$

We see directly that

$$
\left.\left|\varrho_{x}(y)^{-1}-\right| x\right|^{-1} \mid \leqq c_{26} \frac{|y|}{|x||x-y|}
$$

which combined with (3.17), (3.3), and (3.8) shows that

$$
|\omega(x)| \leqq c_{27}\left(1+r^{2}\right)^{-1} \quad \text { for } \quad x \in N_{k} .
$$

To estimate the derivatives of $\omega$ we record the following Schauder estimate whose proof can be found in $[17, \mathrm{p} .161]$. Let $L$ be an elliptic operator on the unit ball of $\mathbb{R}^{3}$ of the form

$$
L u=\sum_{i, j=1}^{3} a_{i j}(\xi) \frac{\partial^{2} u}{\partial \xi^{i} \partial \xi^{j}}+\sum_{j=1}^{3} b_{j}(\xi) \frac{\partial u}{\partial \xi^{j}}+c(\xi) u(\xi)
$$

where $\xi=\left(\xi^{1}, \xi^{2}, \xi^{3}\right)$ is the Cartesian coordinate in the ball. For any function $\varphi(\xi)$ defined on an open set $\Omega$ and real number $\lambda$ with $0<\lambda<1$, define the following norms

$$
\begin{aligned}
& |\varphi|_{0, \Omega}=\sup _{\xi \in \Omega}|\varphi(\xi)| \\
& |\varphi|_{0, \lambda, \Omega}=\sup _{\xi, \bar{\xi} \in \Omega} \frac{|\varphi(\xi)-\varphi(\bar{\xi})|}{|\xi-\bar{\xi}|^{\lambda}} \\
& |\varphi|_{1, \lambda, \Omega}=\sup _{\xi \in \Omega}|\partial \varphi(\xi)|+|\partial \varphi|_{0, \lambda, \Omega} \\
& |\varphi|_{2, \lambda, \Omega}=\sup _{\xi \in \Omega}|\partial \varphi(\xi)|+\left.\sup _{\xi \in \Omega}|\partial \partial \varphi|(\xi)|+| \partial \partial \varphi\right|_{0, \lambda, \Omega} .
\end{aligned}
$$

Let $B_{r}=\{\xi:|\xi|<r\}$. Suppose there is a positive number $\Lambda$ so that

$$
\begin{gathered}
\sum_{i, j=1}\left|a_{i j}\right|_{0, \lambda, B_{1}}+\sum_{i=1}^{3}\left|b_{i}\right|_{0, \lambda, B_{1}}+|c|_{0, \lambda, B_{1}} \leqq \Lambda, \\
\Lambda^{-1}|t|^{2} \leqq \sum_{i, j=1}^{3} a_{i j}(\xi) t^{i} t^{j} \forall t \in \mathbb{R}^{3} \backslash\{0\}, \quad \forall \xi \in B_{1}
\end{gathered}
$$


It then follows that for any $C^{2, \lambda}$ function $u$ on $B_{1}$ we have

$$
|u|_{2, \lambda, B_{1} / 2} \leqq \bar{C}\left(|L u|_{0, \lambda, B_{1}}+|u|_{0, B_{1}}\right)
$$

where $\bar{C}$ depends only on $\lambda, \Lambda$.

We now fix a point $x \in N_{k}$, and assume that $\sigma=\frac{1}{2}\left|x_{0}\right|>\sigma_{0}$. We then let

$$
\xi=\frac{1}{\sigma}(y-x)
$$

where $y$ is our asymptotic coordinate on $N_{k}$. If we let $u(\xi)=\omega(y), a_{i j}(\xi)=g^{i j}(y), b_{k}(\xi)$ $=\sigma g^{i j}(y) \Gamma_{i j}^{k}(y)$, and $c(\xi)=-\sigma^{2} f(y)$ we have

$$
\begin{aligned}
L u(\xi) & =\sum_{i, j} a_{i j}(\xi) \frac{\partial^{2} u}{\partial \xi^{i} \partial \xi^{j}}+\sum_{k} b_{k}(\xi) \frac{\partial u}{\partial \xi^{k}}+c(\xi) u \\
& =\sigma^{2}(\Delta \omega(y)-f \omega(y)) .
\end{aligned}
$$

Now $\Delta \omega-f \omega=A f|y|^{-1}+h-A \Delta|y|^{-1}$ by (3.2) and the definition of $\omega$. From (1.1) we see that

$$
\left.\left.|\Delta| y\right|^{-1}\left|\leqq c_{28}\right| y\right|^{-5}, \quad\left|\partial\left(\Delta|y|^{-1}\right)\right| \leqq c_{29}|y|^{-6}
$$

These together with (3.3) imply

$$
|L u|_{0, \lambda, B_{1}} \leqq c_{30} \sigma^{-3+\lambda} .
$$

It is clear from (1.1) that (3.19) is satisfied for our operator $L u$ with a constant $\Lambda$ independent of $\sigma$. Thus (3.20) gives

$$
|u|_{2, \lambda, B_{1 / 2}} \leqq c_{31}\left(\sigma^{-3+\lambda}+|u|_{0, B_{1}}\right) \text {. }
$$

By (3.18) this gives for any $\lambda, 0<\lambda<1$

$$
|u|_{2, \lambda, B_{1 / 2}} \leqq c_{32} \sigma^{-2} \text {. }
$$

In terms of $\omega$, this implies

$$
\left|\partial \omega\left(x_{0}\right)\right| \leqq c_{32}\left|x_{0}\right|^{-3}, \quad\left|\partial \partial \omega\left(x_{0}\right)\right| \leqq c_{32}\left|x_{0}\right|^{-4} .
$$

This establishes the required growth properties of $\omega$. The expression for $A$ follows by integrating (3.2) over $\left(N \backslash N_{k}\right) \cup\left(N_{k} \cap B_{\sigma}(0)\right)$ using the boundary condition $\frac{\partial v}{\partial \mathbf{n}}=0$ on $\partial N$, and letting $\sigma \rightarrow \infty$.

To prove uniqueness, suppose $\bar{v}$ is another solution of (3.2) satisfying $\bar{v}=O(1 / r)$ and $\frac{\partial \bar{v}}{\partial \mathbf{n}}=0$ on $\partial N$. Then $u=v-\bar{v}$ satisfies

$$
\Delta u-f u=0, \quad u=O(1 / r), \quad \frac{\partial u}{\partial \mathbf{n}}=0 \quad \text { on } \quad \partial N .
$$

We show $u \equiv 0$. Let $\delta>0$ be any number, and let $E_{\delta}=\{x \in N: u(x) \geqq \delta\}$. Because $u$ tends to zero at infinity, we see that $E_{\delta}$ is compact. We multiply (3.21) by $u$ and 
integrate over $E_{\delta}$

$$
\int_{E_{\delta}} u \Delta u=\int_{E_{\delta}} f u^{2}
$$

Integrating by parts and applying (3.21) we have

$$
\begin{aligned}
\int_{E_{\delta}}|D u|^{2} & =-\int_{\partial E_{\delta}}|D u|-\int_{E_{\delta}} f u^{2} \\
& \leqq \int_{E_{\delta}}\left(f_{-}\right) u^{2} \leqq\left(\int_{E_{\delta}} f_{-}^{3 / 2}\right)^{2 / 3}\left(\int_{E_{\delta}} u^{6}\right)^{1 / 3} \\
& \leqq \varepsilon_{0}\left(\int_{E_{\delta}} u^{6}\right)^{1 / 3} .
\end{aligned}
$$

Applying Lemma 3.1 with $\zeta=u-\delta$ on $E_{\delta}, \zeta \equiv 0$ on $N \backslash E_{\delta}$ we have

$$
\left(\int_{E_{\tilde{\delta}}}(u-\delta)^{6}\right)^{1 / 3} \leqq c_{1} \int_{E_{\delta}}|D u|^{2} .
$$

Combining these inequalities and recalling the choice of $\varepsilon_{0}$

$$
\left(\int_{E_{\delta}}(u-\delta)^{6}\right)^{1 / 3} \leqq c_{1} \varepsilon_{0}\left(\int_{E_{\delta}} u^{6}\right)^{1 / 3} \leqq \frac{1}{3}\left(\int_{E_{\delta}} u^{6}\right)^{1 / 3} .
$$

Since $u=O(1 / r)$, we see that $\int_{N} u^{6}<\infty$, so we may let $\delta \rightarrow 0$ and deduce a contradiction unless $u \leqq 0$. Since $-u$ also satisfies (3.21) we must have $u \geqq 0$, so that $u \equiv 0$ on $N$. This concludes the proof of Lemma 3.2.

The next lemma deals with conformal change of metric on $N$.

Lemma 3.3. Suppose $d s^{2}$ is an asymptotically flat metric on $N$ satisfying (1.2). Let $R$ be the scalar curvature function of $d s^{2}$, and suppose $R$ satisfies

$$
\frac{1}{8}\left(\int_{N} R_{-}^{3 / 2}\right)^{2 / 3} \leqq \varepsilon_{0}
$$

where $\varepsilon_{0}$ is defined in Lemma 3.2. Then there is a unique positive function $\varphi$ with $\frac{\partial \varphi}{\partial \mathbf{n}}=0$ on $\partial N$ so that the metric $\overline{d s}^{2}=\varphi^{4} d s^{2}$ is asymptotically flat, scalar flat, and has total mass

$$
\bar{M}=-\frac{1}{32 \pi} \int_{N} R \varphi .
$$

Proof. In order for the metric $\varphi^{4} d s^{2}$ to be scalar flat, the function $\varphi$ must satisfy

$$
\Delta \varphi-\frac{1}{8} R \varphi=0 \text {. }
$$

The function $v=\varphi-1$ then satisfies

$$
\Delta v-\frac{1}{8} R v=\frac{1}{8} R .
$$

In order for $\varphi^{4} d s^{2}$ to be asymptotically flat, $v$ must satisfy the asymptotic conditions of Lemma 3.2. Now Lemma 3.2 applies directly to give a $v$ satisfying (3.23) with $\frac{\partial v}{\partial \mathbf{n}}=0$ on $\partial N$. Thus $\varphi=v+1$ satisfies (3.22) with $\frac{\partial \varphi}{\partial \mathbf{n}}=0$ on $\partial N$. In order 
to prove that $\varphi$ is everywhere positive on $N$, we let $E=\{x \in N: \varphi(x)<0\}$. Since $\varphi$ is asymptotic to one, we see that $\bar{E}$ is compact, and if $E$ is nonempty, we multiply (3.22) by $\varphi$ and integrate by parts on $E$ using $\frac{\partial \varphi}{\partial \mathbf{n}}=0$ on $\partial N$ to obtain

$$
\begin{aligned}
\int_{E}\|D \varphi\|^{2} & =-\frac{1}{8} \int_{E} R \varphi^{2} \\
& \leqq \int_{E}\left(R_{-}\right) \varphi^{2} \\
& \leqq \frac{1}{8}\left(\int_{E} R_{-}^{3 / 2}\right)^{2 / 3}\left(\int_{E} \varphi^{6}\right)^{1 / 3} .
\end{aligned}
$$

Applying Lemma 3.1 to this inequality we have

$$
\left(\int_{E} \varphi^{6}\right)^{1 / 3} \leqq c_{1} \varepsilon_{0}\left(\int_{E} \varphi^{6}\right)^{1 / 3}
$$

which is a contradiction since $\varepsilon_{0} \leqq \frac{1}{3 c_{1}}$. We conclude that $\varphi \geqq 0$ on $N$. That $\varphi>0$ on $N$ now follows from the Hopf maximum principle. The usual proof works directly on the interior of $N$, and the boundary condition $\frac{\partial \varphi}{\partial \mathbf{n}}=0$ allows an easy modification to show $\varphi>0$ on $\partial N$ (see $[17$, p. 61]).

To show that $\partial N$ has positive mean curvature relative to $\bar{d} s^{2}=\varphi^{4} d s^{2}$ we note that if $H$ is the mean curvature function relative to $d s^{2}$ and $\bar{H}$ the mean curvature relative to $\overline{d s}^{2}$, a direct calculation gives

$$
\bar{H}=\frac{1}{\varphi^{2}}\left(H+\frac{4}{\varphi} \frac{\partial \varphi}{\partial \mathbf{n}}\right)=\frac{1}{\varphi^{2}} H,
$$

so $\bar{H}>0$. This finishes the proof that $\overline{d s}^{2}$ is an asymptotically flat metric on $N$. The formula for $\bar{M}$ follows from Lemma 3.2 .

A special case of Lemma 3.3 is the following corollary which was proved by O'Murchadka and York [19] in case $N$ is diffeomorphic to $\mathbb{R}^{3}$.

Corollary 3.1. If $M=0, R \geqq 0$, and $R$ is not identically zero, then there is a metric conformally equivalent to $d s^{2}$ which is asymptotically flat, scalar flat, and so that $N_{k}$ has negative total mass.

Theorem 1 and Corollary 3.1 imply that an asymptotically flat metric satisfying the hypotheses $M=0, R \geqq 0$ must have $R \equiv 0$ on $N$. We assume now that $d s^{2}$ is such a metric and that (1.2) is also satisfied. We define a one-parameter family of metrics $d s_{t}^{2}$ on $N$ by

$$
d s_{t}^{2}=\sum_{i, j=1}^{3}\left(g_{i j}+t S_{i j}\right) d x^{i} d x^{j}
$$

where $S_{i j}$ is the Ricci tensor of $d s^{2}$. These metrics are defined in a neighborhood of $t=0$ by (1.1) and (1.2), and $d s_{0}^{2}=d s^{2}$. For $t$ sufficiently small, $d s_{t}^{2}$ is asymptotically 
flat by (1.2) and because $\partial N$ has positive mean curvature relative to $d s^{2}$, so by continuity $\partial N$ also has positive mean curvature relative to $d s_{t}^{2}$ for $t$ small. Let $R_{t}$ be the scalar curvature function of $d s_{t}^{2}$, so that we have $R_{0}=R \equiv 0$. A known formula (see [20]) gives

$$
R_{0}^{\prime}=\left.\frac{d}{d t} R_{t}\right|_{t=0}=-\Delta R+\delta \delta \mathrm{Ric}-\|\mathrm{Ric}\|^{2}
$$

where $\operatorname{Ric}=\left(S_{i j}\right)$ is the Ricci tensor, and

$$
\partial \partial \mathrm{Ric}=S_{|i| j \mid}^{i j},\|\mathrm{Ric}\|^{2}=g^{i k} g^{j l} S_{i j} S_{k l} .
$$

Since $R \equiv 0$, we have $\Delta R \equiv 0$, and a direct application of the second Bianchi identity shows

$$
\delta \delta \text { Ric }=2 \Delta R \equiv 0 .
$$

Thus (3.24) becomes

$$
R_{0}^{\prime}=-\|\operatorname{Ric}\|^{2} \text {. }
$$

Since $R_{0} \equiv 0$, it follows from (1.2) that for $t$ sufficiently small we have

$$
\frac{1}{8}\left(\int_{N} R_{t-}^{3 / 2}\right)^{2 / 3} \leqq \varepsilon_{0}
$$

where $\varepsilon_{0}$ can be taken independent of $t$ for small $t$. Applying Lemma 3.3, we find a function $\varphi_{t}$ so that the metric $\varphi_{t}^{4} d s_{t}^{2}$ is asymptotically flat and scalar flat. The mass $M(t)$ of this metric is

$$
M(t)=-\frac{1}{32 \pi} \int_{N} R_{t} \varphi_{t} \sqrt{g_{t}} d x
$$

where $\sqrt{g_{t}}$ is the volume factor for $d s_{t}^{2}$.

We will prove that $\frac{d M}{d t}$ exists at $t=0$, and can be computed by differentiating (3.26) under the integral sign. For small $h$, let $\varphi^{(h)}$ be defined by

$$
\varphi^{(h)}=\frac{\varphi_{h}-\varphi_{0}}{h} .
$$

Let $\Delta_{t}$ be the Laplacian for the metric $d s_{t}^{2}$, and let $\Delta^{(h)}$ be the differential operator defined by

$$
\Delta^{(h)} v=\frac{1}{h}\left(\Delta_{h} v-\Delta_{0} v\right)
$$

Let $R^{(h)}=\frac{1}{h}\left(R_{h}-R_{0}\right)$. The function $\varphi^{(h)}$ satisfies the equation

$$
\Delta_{0} \varphi^{(h)}-\frac{1}{8} R_{0} \varphi^{(h)}=-\Delta^{(h)} \varphi_{h}+\frac{1}{8} R^{(h)} \varphi_{h}
$$

By (1.1) and (1.2), we see that this equation satisfies the hypotheses of Lemma 3.2. Since $\varphi^{(h)}$ is $O(1 / r)$, that lemma implies

$$
\left|\varphi^{(h)}\right| \leqq \gamma_{1}(1+r)^{-1} \quad \text { on } \quad N_{k}
$$


where $\gamma_{1}$ is independent of $h$. Standard linear theory applied to (3.27) shows that $\varphi^{(h)}$ has a local $C^{2, \alpha}$ bound depending on $C^{1}$ bounds on $R_{0}$ and $-\Delta^{(h)} \varphi_{h}+\frac{1}{8} \mathrm{R}^{(h)} \varphi_{h}$. Since these bounds are independent of $h$, we can find a sequence $\left\{h_{i}\right\}$ tending to zero so that $\varphi_{h_{\imath}}$ converges in $C^{2, \beta}$ norm for any $\beta<\alpha$ uniformly on compact subsets of $N$ to a $C^{2, \alpha}$ function $\varphi_{0}^{\prime}$ which satisfies

$$
\Delta_{0} \varphi_{0}^{\prime}-\frac{1}{8} R_{0} \varphi_{0}^{\prime}=-\Delta_{0}^{\prime} \varphi_{0}+\frac{1}{8} R_{0}^{\prime} \varphi_{0}
$$

where

$$
\Delta_{0}^{\prime}=\left.\frac{d}{d t} \Delta_{t}\right|_{t=0}, \quad \text { and } \quad R_{0}^{\prime}=\left.\frac{d}{d t} R_{t}\right|_{t=0} .
$$

By (3.28) we have $\varphi_{0}^{\prime}=O(1 / r)$ so the uniqueness part of Lemma 3.2 implies that the limit $\varphi_{0}^{\prime}$ is independent of the sequence $\left\{h_{i}\right\}$ we have chosen. Thus it follows that $\frac{d}{d t} \varphi_{t}$ exists at $t=0$ and is equal to $\varphi_{0}^{\prime}$. From (1.1) and (1.2) we have constants $\gamma_{2}, \gamma_{3}$ independent of $h$ so that

$$
\left|R^{(h)}\right| \leqq \gamma_{2}\left(1+r^{3+\alpha}\right)^{-1} g^{(h)}=\gamma_{3}\left(1+r^{1+\alpha}\right)^{-1} \quad \text { on } \quad N_{k}
$$

where $g^{(h)}=\frac{1}{h}\left(g_{h}-g_{0}\right)$. We now apply (3.28), (3.29), and the dominated convergence theorem to conclude that $M^{\prime}(0)=\left.\frac{d}{d t} M(t)\right|_{t=0}$ exists and

$$
M^{\prime}(0)=-\frac{1}{32 \pi} \int_{N} R_{0}\left(\varphi_{t} \sqrt{g_{t}}\right)^{\prime} d x-\frac{1}{32 \pi} \int_{N} R_{0}^{\prime} \varphi_{0} \sqrt{g_{0}} d x .
$$

Since $R_{0} \equiv 0$ and $\varphi_{0} \equiv 1$, we may apply (3.25) to conclude

$$
M^{\prime}(0)=\frac{1}{32 \pi} \int_{N}\|\mathrm{Ric}\|^{2} .
$$

If Ric is not identically zero, (3.30) implies that $M^{\prime}(0)>0$ and hence by choosing a suitable $t_{0}<0$ we. would have $M\left(t_{0}\right)<0$. The metric $\varphi_{t_{0}}^{4} d s_{t_{0}}^{2}$ would then be asymptotically flat, scalar flat, and $N_{k}$ would have negative total mass in contradiction to Theorem 1 . Hence we conclude that Ric $\equiv 0$, and because we are working in dimension three, $d s^{2}$ is flat. This completes the proof of Theorem 2.

\section{Appendix}

In this appendix we give a brief discussion of the Regularity Estimate (2.1), and the existence of smooth solutions of the two-dimensional problem of least area for surfaces (regardless of topological type) having a given boundary curve in a Riemannian 3-manifold with boundary of positive mean curvature. These results are well-known so we mainly give references and briefly indicate a few of the simpler arguments involved. The (interior) Regularity Estimate (2.1) and the existence theorem are part of the powerful approach to minimal surfaces which has developed through the use of geometric measure theory. A thorough account 
of this field is given by Federer [16] where Chapter 5 discusses the applications to variational problems of area type. We refer the reader to the introduction to that chapter for an account of the people involved in the developments of this theory. The Regularity Estimate (2.1) can be extracted as a very special case of the material in Section 5.3 and Theorem 5.4 .15 of [16]. A more differential geometric approach to this estimate can be found in [21].

The existence of an area minimizing current $S_{\sigma}$ (surface with singularities) having boundary curve $C_{\sigma}$ follows from 5.1 .6 of [16]. The above mentioned regularity theory implies that $S_{\sigma}$ has no singularities in the interior of $N \cap S_{\sigma}$. We will show that the positive mean curvature of $\partial N$ implies that $S_{\sigma}$ lies entirely in the interior of $N$, and hence $S_{\sigma} / C_{\sigma}$ is completely regular. To see this, consider a boundary component $B$ of $N$. For $\varepsilon>0$ sufficiently small, the open set $\mathcal{O}_{t}=\{x \in N$ : $\operatorname{dist}(x, B)<t\}$ for $0<t<\varepsilon$ retracts smoothly onto $B$, and the parallel surfaces $B_{t}=\{x \in N: \operatorname{dist}(x, B)=t\}$ for $0 \leqq t<\varepsilon$ are smooth surfaces diffeomorphic to $B=B_{0}$. Let $v$ be the outward unit normal vector field to $B$, and for $0<t<\varepsilon$, extend $v$ as a vector field on $\mathcal{O}_{t}$ by parallel translation along geodesics normal to $B$. Thus on $B_{t}, v$ is a unit normal vector field. The fact that $B$ has positive mean curvature with respect to $v$ says that $\operatorname{div}(v)>0$ on $B$ where $\operatorname{div}(\cdot)$ is divergence of a vector field taken on $N$. By continuity we then have

$$
\operatorname{div}(v)>0 \text { on } \mathcal{O}_{t}
$$

for some $t \in(0, \varepsilon)$. The theory of Chapter 5 of [16] also gives us an open set $V_{\sigma} \subseteq N$ so that

$$
S_{\sigma}=\partial V_{\sigma}
$$

in the sense of geometric measure theory. Let $\eta$ be the outward (to $V_{\sigma}$ ) pointing unit normal vector field of $S_{\sigma}$ (which exists almost everywhere with respect to Hausdorff 2-dimensional measure), and apply the divergence theorem, Theorem 4.5.6 of [16] to the open set $\mathcal{O}_{t} \cap V_{\sigma}$. If this set is not empty we have

$$
\int_{S_{\sigma} \cap \mathcal{O}_{t}}\langle\eta, v\rangle d \mathscr{H}^{2}-\int_{B_{t} \cap V_{\sigma}} 1 d \mathscr{H}^{2}>0
$$

where $\mathscr{H}^{2}$ is Hausdorff 2-dimensional measure on $N$. This implies Area $\left(B_{t} \cap V_{\sigma}\right)$ $<$ Area $\left(S_{\sigma} \cap \mathcal{O}_{t}\right)$ contradicting the area minimizing property of $S_{\sigma}$. This shows that $S_{\sigma}$ lies strictly away from $\partial N$ as claimed.

Acknowledgement. We would like to thank Professor D. Brill, S. S. Chern, R. Geroch, A. Lichnerowicz, J. Marsden and I. M. Singer for their interest in this work.

\section{References}

1. Arnowitt, R., Deser, S., Misner, C.: Phys. Rev. 118, 1100 (1960 b)

2. Geroch, R.: General Relativity. Proc. Symp. Pure Math. 27, 401-414 (1975)

3. Brill, D., Deser, S. : Ann. Phys. 50, 548 (1968)

4. Choquet-Bruhat, Y., Fischer, A., Marsden, J.: Maximal hypersurfaces and positivity of mass. Preprint (1978)

5. Choquet-Bruhat, Y., Marsden, J.: Solution of the local mass problem in general relativity. Commun. math. Phys. 51, 283-296 (1976) 
6. Jang, P. S. : J. Math. Phys. 1, 141 (1976)

7. Leibovitz, C., Israel, W.: Phys. Rev. 1 D, 3226 (1970)

8. Misner, C.: Astrophysics and general relativity, Chretien, M., Deser, S., Goldstein, J. (ed.). New York: Gordon and Breach 1971

9. Geroch, R. : J. Math. Phys. 13, 956 (1972)

10. Chern, S.S.: Minimal submanifolds in a Riemannian manifold. University of Kansas (1968) (mimeographed lecture notes)

11. Finn, R.: On a class of conformal metrics, with application to differential geometry in the large. Comment. Math. Helv. 40, 1-30 (1965)

12. Huber, A.: Vollständige konforme Metriken und isolierte Singularitäten subharmonischer Funktionen. Comment. Math. Helv. 41, 105-136 (1966)

13. Huber, A.: On subharmonic functions and differential geometry in the large. Comment. Math. Helv. 32, 13-72 (1957)

14. Huber, A.: On the isoperimetric inequality on surfaces of variable Gaussian curvature. Ann. Math. 60, 237-247 (1954)

15. Alexander, H., Osserman, R.: Area bounds for various classes of surfaces. Am. J. Math. 97 (1975)

16. Federer, H.: Geometric measure theory. Berlin, Heidelberg, New York: Springer 1969

17. Morrey, C.B.: Multiple integrals in the calculus of variations. Berlin, Heidelberg, New York: Springer 1966

18. Hörmander, L.: Linear partial differential operators. Berlin, Heidelberg, New York : Springer 1969

19. O’Murchadha, N., York, J.W.: Gravitational Energy. Phys. Rev. D 10, 2345-2357 (1974)

20. Kazdan, J., Warner, F.: Prescribing curvatures. Proc. Symp. Pure Math. 27, 309-319 (1975)

21. Schoen, R., Simon, L., Yau, S.-T. : Curvature estimates on minimal hypersurfaces. Acta Math. 134, 275-288 (1975)

Communicated by R. Geroch

Received September 5, 1978 